\title{
A framework for the provision of flexibility services at the transmission and distribution levels through aggregator companies
}

\author{
Ioannis Lampropoulos* , Tarek Alskaif, Jelle Blom, Wilfried van Sark \\ Energy \& Resources Group, Copernicus Institute of Sustainable Development, Utrecht University, Princetonlaan 8a, 3584 CB Utrecht, The Netherlands
}

\section{A R T I C L E I N F O}

\section{Article history:}

Received 31 January 2018

Received in revised form 6 December 2018

Accepted 30 December 2018

Available online 4 January 2019

\section{Keywords:}

Demand-side management

Flexibility services

Market optimisation

Ancillary services

Aggregation entities

Real-time systems

\begin{abstract}
A B S T R A C T
A hierarchical control framework is proposed that enables the provision of flexibility services in power systems through aggregation entities. The focus is on both wholesale energy trade functions, i.e. dayahead market optimisation, and ancillary services to the operators of the transmission and distribution systems, i.e. provision of automatic frequency restoration reserves and peak shaving services. The control framework is generic, scalable, and is effectively configured to address all those diverse needs originating from the lower level, i.e. the device or the user, to the distribution and transmission levels. The work includes the establishment of procedures to identify and solve possible conflicts between the operators of the transmission and distribution systems when procuring ancillary services with competing objectives. The framework is applied on a case study focusing on an aggregation of residential buildings in the Netherlands which are equipped with photovoltaic installations and battery-based energy storage systems. The annual costs and benefits are calculated by using historical market data, whereas the simulation scenarios address the impact of different levels of generation and demand forecasting errors in the economic performance. The outcome of computer simulations provides an informative insight into the differences between the strategies of aggregator companies, and the potential of the investigated markets. The economic evaluation includes the value estimation of peak shaving services at the distribution level. An increase in procurement costs for such services is expected to stimulate the distribution system operators to invest into grid capacity enhancement.
\end{abstract}

(c) 2019 Elsevier Ltd. All rights reserved.

\section{Introduction}

Recent developments in distribution grids, environmental policy, and the energy market liberalisation process, have resulted in a quest for flexibility in the operation of power systems. Flexibility is defined as a "general" concept of elasticity of resource deployment providing ancillary services for the grid stability and/or market optimisation" [1]. In this paper, a hierarchical control framework is proposed to exploit the flexibility, which is inherent in an aggregation of small-scale and/or medium-scale system users, for both market optimisation purposes and the provision of ancillary services. To achieve this, the control framework has to be effectively configured to address all those diverse needs originating from the lower level, i.e. the device or the user, to the global level, i.e. the power system.

Several control schemes have been proposed in the literature for enabling flexibility from demand-side resources [2]. Centralised approaches have been proposed for the management of groups of homogeneous distributed resources, e.g. space heaters

\footnotetext{
This research did not receive any specific grant from funding agencies in the public, commercial, or not-for-profit sectors.

* Correspondence to: Princetonlaan 8a, 3584 CB Utrecht, The Netherlands.

E-mail address: i.lampropoulos@uu.nl (I. Lampropoulos).
}

[3], and electric vehicles (EVs) [4]. Though, centralised approaches are impractical for a large aggregation of devices, and encounter both scalability limitations and privacy concerns, whereas direct load control schemes might also interfere with the user comfort level [5]. Contrary to centralised control schemes, the management of distributed resources could theoretically be addressed through decentralised approaches, for example by enabling distributed resources to operate as frequency responsive resources [6-8], as in the context of frequency regulation provided by generation units through droop control. Such decentralised control schemes could address the operation of autonomous systems operated in islanded mode, but it becomes impossible to carry all necessary control actions within a large interconnected power system solely based on frequency [5].

Considering the special characteristics of small-scale system users such as residential customers, and prosumers ${ }^{1}$ [9], who are in principle non-professionals seeking for a protective pricing environment and means to avoid high risks, the concept of voluntary participation in demand side management (DSM) appears to be a

\footnotetext{
1 Prosumer is a portmanteau derived by combining the word professional (or producer, or provider) with the word consumer.
} 
rather attractive option. Price-based control approaches involve indirect load control in the form of differentiated retail pricing structures, and reflect a voluntary nature, however, such approaches might result in a rather uncertain aggregate response as a consequence of differing users' objectives and employed technology [5]. Previous work on price-based schemes has been focused on utilising demand response (DR) as an hourly energy resource [10,11]. However, the challenge is to shape the demand for electricity to support fast regulation. The application of a price-based control scheme with a shorter time resolution, e.g. on a minute basis, will most probably be opposed by residential customers, since they will have to adjust their activities within this narrow timescale.

Software agent platforms have been proposed for the marketbased participation of distributed resources in wholesale and ancillary services markets [12]. A general notion of the agent concept attributes a degree of autonomy to agents, and attaches importance to their reactive characteristics in response to changes within their environments [13]. A software agent can represent a process, or a group of processes, and utilise internal models to optimise certain quantities over a prediction horizon within certain constraints. At the customer side, the agent resides within a Home Energy Management System (HEMS) that integrates sensors and meters for monitoring purposes, optimisation and control. The incorporation of a prediction horizon for optimisation purposes within the HEMS is particularly useful, especially considering the variability related to intermittent renewable energy sources (RES). Apparently, it becomes essential with respect to future practical applications to devise methods for the management of distributed resources that reflect the global objectives of power system control while allowing for distributed control and computation. In this context, hierarchical control structures are considered as indispensable paradigms for the management of distributed resources [14].

In this paper, the focus is on the hierarchical management of distributed resources for the provision of system services through intermediaries, i.e. aggregator companies, ${ }^{2}$ and three types of decomposition are considered which are essential in electrical power systems, i.e. level, time and mode decomposition [15]. A generic and scalable framework is proposed that enables the provision of flexibility in power systems operations, from demandside resources, through aggregators. It is generic because it considers a uniform representation of non-homogeneous distributed resources based on their characteristics of energy utilisation, and not on their physical specifications or operational functions, compared to other proposed frameworks and models that focus on the management of homogeneous types of demand-side resources such as EVs $[16,17]$. It is scalable because it is based on a hierarchical level structure characterised by decentralised decision making and distributed computation. Emphasis is given on both wholesale energy trade functions and the provision of ancillary services to the system such as fast operating reserves that can be deployed on a minute-to-minute basis, and local network support. Previous work has been focused on the interaction of aggregators with wholesale spot markets, and Transmission System Operators (TSOs), i.e. the operators of high voltage (HV) grids, for the provision of ancillary services such as tertiary upward regulation reserve capacity [18], and secondary upward-downward regulating reserve capacity [17, 19]. However, in all these works, the interactions with Distribution System Operators (DSOs) for the provision of local network support have not been addressed. With the transition to more decentralised power systems, and the increasing integration of distributed resources in Low Voltage (LV) grids, the focus of recent research is increasingly placed on the interactions of multiple aggregators with DSOs during flexibility activation events for short-term congestion

\footnotetext{
2 Also called aggregation service providers but referred to as simply 'aggregator' in the remaining part of this paper.
}

management in distribution networks [16,20,21]. In this paper, the former ideas presented in [19] are further developed to address the participation of multiple aggregators, as well as the provision of local network support functions as considered from the perspective of DSOs. In this context, it is highly relevant to consider the establishment of procedures to identify and solve possible conflicts between the TSO and the DSO that take place when requesting and/or procuring ancillary services with competing objectives [22]. This is in accordance with the EU regulation 2017/1485, where it is stated that "TSOs and DSOs shall cooperate in order to facilitate and enable the delivery of active power reserves by reserve providing groups or reserve providing units located in the distribution systems" [23]. In [16], the authors proposed a framework for the information characterisation between aggregators, system operators and users by presenting the conceptual basis for defining the aggregator role in the electricity market. In this paper, certain conditions are distinguished to ensure the efficient utilisation of flexibility services sourced from distributed resources, when congestion occurs at the distribution level.

The main contribution in this paper is the definition of a hierarchical control framework which captures the a priori operational planning phase, the real-time operations, as well as the a posteriori financial settlement. As part of the proposed framework, a forecast-based optimisation tool is developed to address wholesale electricity trade functions. Although the framework is applied on a case study focusing on the Netherlands, it can be applied to other countries as well. Annual costs and benefits are calculated by using historical market data. Earlier work on the economic optimisation of distributed energy storage, coupled with residential demand and PV generation, addressed a revenue analysis of the passive contribution in system balancing which is a voluntary scheme attributable to the Dutch system organisation [24,25]. In this paper, the focus of the economic analysis is on the active contribution in system balancing, i.e. the provision of automatic Frequency Restoration Reserves (aFRR). Furthermore, as part of the economic analysis in [24] and [25], the electricity costs and potential revenues were computed under the assumption of accurate predictions, by utilising historical market data. However, in a real-life application, forecast errors will inevitably occur and the potential revenues will always be dependent on the accuracy of the forecasting methods. To address the impact of forecasting errors on the economic performance, different simulation scenarios were developed for this study, which are differentiated on the basis of different levels of forecast error in PV generation and demand. Finally, another novelty in this paper is the value estimation of peak shaving services at the distribution level, by associating local network support functions to the procurement of balancing reserves.

The paper is structured as follows: Section 2 provides a description of the system design and the hierarchical framework. In Sections $3-5$, the operational planning, the real-time operation, and the settlement phases are elucidated respectively. In Section 6 the case study is presented, whereas the simulation results are presented and analysed in Section 7. The paper ends with discussion and conclusions.

\section{The system design and the hierarchical framework}

In this work, three main groups of actors are distinguished: the system operators (including the operators of the electricity markets, and the transmission and distribution systems), the aggregators (legal entities that hold contracts with system users for the provision of energy and ancillary services), and the system users, i.e. producers and consumers. ${ }^{3}$ Each $j$ th user, with $j=1$,

\footnotetext{
3 Simply referred as 'users' or 'residential users' in the remaining part of this paper.
} 


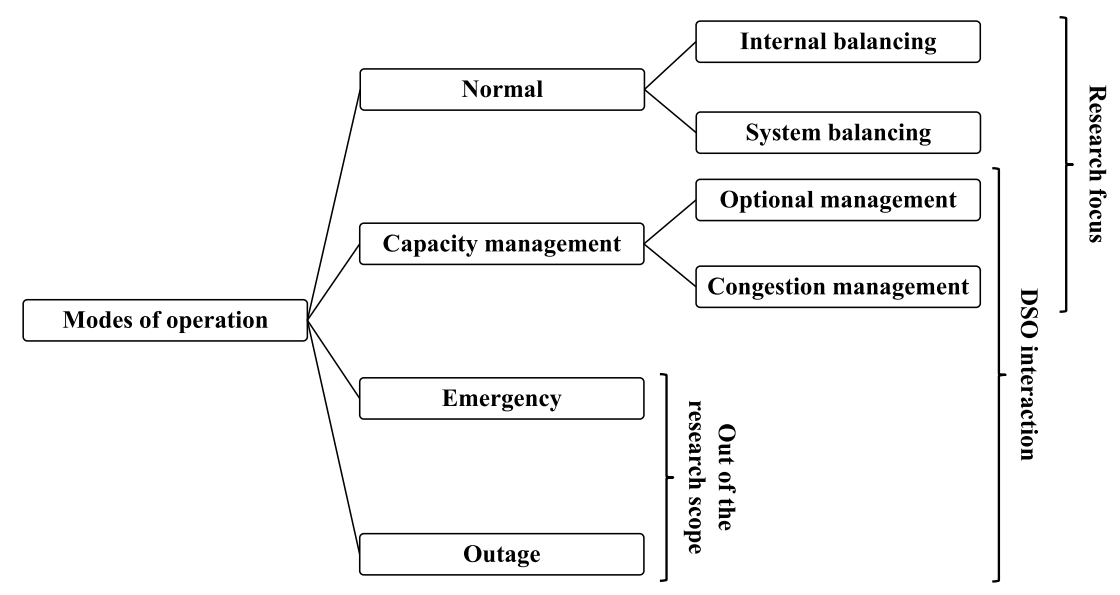

Fig. 1. Hierarchical scheme of the system modes of operation, indicating the research focus and the perspective of the DSO.

$\ldots, N$, is associated with a single aggregator $a$, with $a=1, \ldots, A$. Regional DSOs connect the individual users to the grid and provides the distribution of electricity. Medium voltage (MV) electrical networks are connected to LV networks through MV/LV transformer substations, which subsequently feed a large number of users at the LV level. In line with the hierarchical level structure of power systems, the information associated with a number of users is aggregated first at the MV/LV substation and subsequently at the HV/MV substation. This ensures that both the DSO and the TSO are aware of available resources that can be activated at each level to perform their tasks. Then, single-sided markets can be organised at each substation level where the aggregators can place aggregate bids originating from their associated users. An aggregator can undertake different roles in the electricity market such as the role of the Balance Responsible Party (BRP), the role of the supplier, but can also provide ancillary services to the grid operators. The existence of the users' agents at the users' premises entails two advantages; firstly, decisions can also be taken locally in a decentralised manner, and secondly, distributed computation can be achieved. Furthermore, the models, objectives and constraints can be revised through software updates, ensuring that the proposed design will not become obsolete in the future due to unforeseen developments.

\subsection{Modes of operation}

In Fig. 1, the hierarchical system modes of operation are depicted. In normal mode (i.e. the power system is in balance), the aggregator and its associated system users are acting to maintain their internal balance, whereas in the case of system imbalances they are collectively contributing to system balancing. Internal imbalances occur at the portfolio level of each aggregator and refer to deviations from their energy schedules and/or contracts, whereas system balancing is organised by the TSO. In normal operations, i.e. no congestions are expected at the distribution level, the DSO does not undertake any actions. In the case that congestions arise, i.e. capacity management mode, the DSO can procure flexibility products for congestion management, e.g. through a local single buyer market, to maintain the power profiles within the operating limits of network assets such as transformers and cables, or can take preventive actions, i.e. optional capacity management. The optional capacity management mode arises when certain grid assets, such as a MV/LV transformer, are expected to get overloaded, based on predictions, in the case that the TSO requests operating reserves for frequency regulation (see Section 4.1). This is in line with the EU regulation 2017/1485, where it is specified that "Each reserve connecting DSO and each intermediate DSO shall have the right, in cooperation with the TSO, to set, before the activation of reserves, temporary limits to the delivery of active power reserves located in its distribution system" [23]. The modes of emergency and outage are outside the scope of this paper and are considered for future work. According to the schematic in Fig. 1, the modes of operation are prioritised as follows: congestion management (if congestion occurs at the LV feeders and transformer), system balancing (organised by the TSO), and finally internal balancing of the aggregator portfolio. The HEMS of users will act first to address the local problems, i.e. congestion management at the distribution level. The logic is that local problems should primarily be addressed by the resources that are located close to the fault occurrence, whereas global challenges (system imbalances) can be addressed by all system resources.

\subsection{System model and constraints}

The aggregate electricity net supply and demand of a user can be distinguished between a non-controllable part, i.e. any consuming and/or generating device which cannot be controlled, and a flexible/controllable part, i.e. any consuming and/or generating device which can be controlled in a flexible manner. In this work, since bidirectional power flows are considered, by convention it is assumed that power values are positive for the demand figures, and negative for generation figures. The power exchange with the grid of the $j$ th user at time instant $t$ is as follows:

$P_{j}(t)=P_{j}^{\text {base }}(t)+P_{j}^{\text {flex }}(t), \forall t$

where $P_{j}^{\text {base }}(t)$ is the base load (non-controllable net supply and demand), and $P_{j}^{\text {flex }}(t)$ is the flexible load and/or generation at time $t$. The constraint regarding the power exchange with the grid of the jth user can be written as follows:

$P_{j, \min } \leq P_{j}(t) \leq P_{j, \max }, \forall t$,

where $P_{j, \max }$, and $P_{j, \min }$ are constant values defined as power boundaries for each $j$ th user at the point of connection to the grid. Grid assets, such as transformers and cables, are also characterised by power exchange constraints. For simplicity purposes, the paper descriptions address one LV distribution grid and the interactions with a single DSO, however, the proposed framework can be well expanded to address aggregators interacting with several DSOs and users connected to different MV and LV grids. Considering a distribution grid where each $j$ th user is fed by a single transformer, the absolute sum of net power supply and demand of all users connected to that transformer must be maintained within its 
operating limits, i.e. the transformer capacity constraint $P_{\max }^{\text {trans }}$ :

$\left|\sum_{j=1}^{N} P_{j}(t)\right| \leq P_{\max }^{\text {trans }}$

In the following, the above mentioned constraints are framed next to the optimisation sub-problems during the operational planning (See Section 3.), and the real-time operation (see Section 4.).

\section{Operational planning phase}

\subsection{Energy trade}

During the a priori operational planning, each aggregator $a$ determines an energy schedule, i.e. a piecewise function with a finite value for each settlement period denoted as $h=1, \ldots, H$ with $H=24 \mathrm{~h}$. The energy schedule $E_{a}^{e s}(h)$ of aggregator $a$ is constructed based on individual hourly contributions that are provided from its associated users, and is defined as follows:

$$
\begin{aligned}
E_{a}^{e s}(h) & =\sum_{j \in a} E_{j}^{e s}(h)=\sum_{j \in a} P_{j}^{\text {das }}(h) \cdot \tau_{h} \\
& =\sum_{j \in a}\left(P_{j}^{\text {das, base }}(h)+P_{j}^{\text {das,flex }}(h)\right) \cdot \tau_{h}, \forall h
\end{aligned}
$$

where $E_{j}^{e s}(h)$ is the energy schedule of the $j$ th user at the $h$ th hour, $P_{j}^{\text {das }}(h)$ is the day-ahead power schedule based on Eq. (1), and $\tau_{h}$ is the planning period which is equal to $1 \mathrm{~h}$. A constraint bounded linear optimisation approach is utilised to minimise the energy cost for the $j$ th user through energy arbitrage in the day-ahead market (DAM). The optimisation model is derived from an earlier developed model for the maximisation of self-consumption [26, 27]. Within the context of a multi-revenue streams business model, an aggregator shall implement a strategy for determining the allocation of its portfolio flexibility to different applications such as spot market optimisation and the provision of ancillary services. A decision for the optimal strategy is dependent on the portfolio technical characteristics, the economic potential of a certain application as well as the associated risks. Even though it is not within the scope of this paper to determine the optimal allocation of flexibility, two different strategies, based on heuristics, are tested as part of the investigated case study and the developed scenarios (see Section 6.1). Assuming certain predictions of local generation and demand, and the market clearing prices, the constrained optimisation problem is formulated as the cost minimisation over a finite horizon of $24 \mathrm{~h}$ :

$\min _{P_{j}^{\text {das }, f l e x}(h)}\left\{\sum_{h=1}^{H} P_{j}^{\text {das,flex }}(h) \cdot \pi^{\text {prd }}(h)\right\}$

where $P_{j}^{\text {das,flex }}(h)$ represents the optimisation parameter, i.e. the optimised day-ahead power schedule of the $j$ th user, and $\pi^{p r d}(h)$ represents a prediction of the DAM price. The day-ahead optimisation problem is subject to the power constraint regarding the power exchange with the grid (see Eq. (2)):

$P_{j, \text { min }} \leq P_{j}^{\text {das, base }}(t)+P_{j}^{\text {das, flex }}(t) \leq P_{j, \max }, \forall t$

In the case that the submitted energy schedule $E_{a}^{e s}(h)$ is cleared at the market, then it is automatically transformed into an energy contract $E_{a}^{e c}(h):=E_{a}^{e s}(h), \forall h, a$, which dictates the power transfer with the power system during the actual delivery day. An energy contract represents the energy volumes which have been cleared in the market on behalf of aggregator $a$, either through long-term (forward and future markets) or short-term trade (spot markets), i.e. the sum of the non-controllable part of the net supply and demand, and the optimised aggregate schedule of flexible resources.

\subsection{Provision of ancillary services}

For the case of the $j$ th user, the energy capabilities for the provision of ancillary services, i.e. upwards and downwards regulation, correspond to the minimum and maximum energy demand that can be achieved at each hour $h$ by its flexible resources. Note that the availability of energy capabilities are subject to the commitments made $a$ priori (e.g. the optimised day-ahead planning), the technical constraints, the users' preferences and the flexibility services provided in the past. The amount of flexibility services provided in the past affect the energy capabilities that can be provided in the future, thus the energy capabilities should be updated regularly. For example, in the case that a bid corresponding to the $h$ th settlement period is activated, then the bids in consequent settlement periods will be affected. In this study, the energy capabilities are updated on an hourly basis. The first step is about calculating the available capacity per user connection:

$P_{j}^{\text {grid,up }}(h)=P_{j, \max }-P_{j}^{\text {das }}(h)$

$P_{j}^{\text {grid,down }}(h)=P_{j, \min }-P_{j}^{\text {das }}(h)$

where $P_{j}^{\text {grid,up }}(h)$ and $P_{j}^{\text {grid,down }}(h)$ represent the available capacity at the connection of the $j$ th user for up- and down-regulation respectively. Subsequently, each $j$ th user shall determine the available capacity for upwards $P_{j}^{\text {flex,up }}(h)$ and downwards $P_{j}^{\text {flex,down }}(h)$ regulation of its flexible resources given any technical constraints and subject to commitments that are made a priori (for an example of this process the reader is advised to check the case study descriptions in Section 6.3, and specifically Eqs. (41)-(42)). Eventually, the energy capabilities of the $j$ th user for upwards and downwards regulation at hour $h$ are determined as follows:

$P_{j}^{u p}(h)=\min \left(P_{j}^{\text {grid,up }}(h), P_{j}^{\text {flex }, \text { up }}(h)\right)$

$P_{j}^{\text {down }}(h)=\min \left(P_{j}^{\text {grid,down }}(h), P_{j}^{\text {flex,down }}(h)\right)$

Each aggregator $a$ collects information from its associated users, and calculates the aggregate energy capabilities for flexibility services. These capabilities are represented as the sum of the hourly power figures for upwards and downwards regulation of all users associated with aggregator $a$ :

$P_{a}^{u p}(h)=\sum_{j \in a} P_{j}^{u p}(h)$

$P_{a}^{\text {down }}(h)=\sum_{j \in a} P_{j}^{\text {down }}(h)$

An aggregator should implement a strategy for transforming the energy capabilities into market bids for operating reserves. In [28], the authors suggested that by taking into account the probability of reserves to be dispatched, an aggregator can select an adequate number of households' availability to construct bids for replacement reserves with a lower risk and a higher expected value. The strategy of an aggregator should aim in the efficient utilisation of the aggregate flexible resource, e.g. the aggregator should not deplete its flexible resources without making a financial gain, and/or the aggregator may averse risk by reserving a part of the flexible resources for internal balancing purposes. A bid should also be accompanied by a price for which an aggregator is willing to provide flexibility to the system operators. 


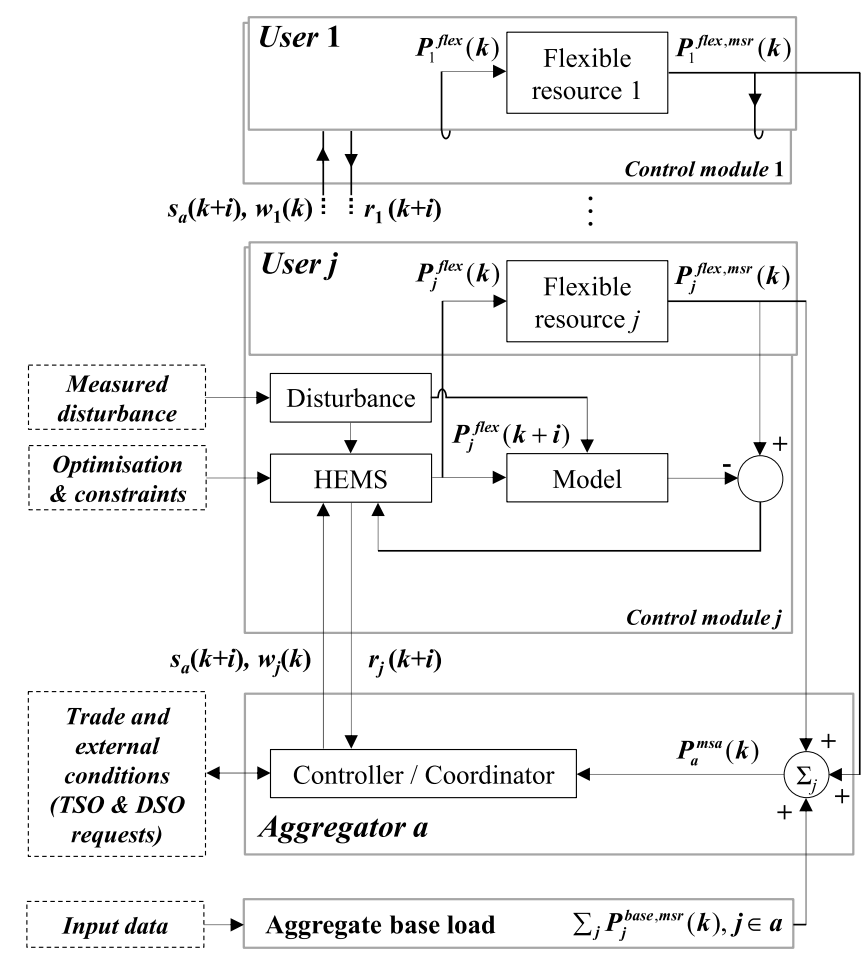

Fig. 2. The simulation model illustrating the real-time operations at time instant $k$. The $j$ th user is illustrated together with the control module block.

\section{Real-time operations}

In the proposed discrete time control procedure, the time interval for simulations and for sampling analogue measurements is set to $1 \mathrm{~min}$., i.e. a $24 \mathrm{~h}$ period corresponding to 1440 discrete time control periods, whereas the current imbalance settlement period (ISP) is denoted as $q \in[1, L]$, with each ISP containing $15 \mathrm{~min}$. corresponding to $l=1, \ldots L$ with $L=96$ per day. Note that the real-time optimisation problem is solved over a decreasing horizon with an endpoint at the end of the qth settlement period.

In real-time operations each aggregator $a$ has to comply with the a priori defined energy contract $E_{a}^{e c}(l)$, whereas in the case that the TSO and/or the DSO request the provision of ancillary services, that aggregator coordinates its associated users to provide the requested services in a collective manner. The proposed control scheme is based on feedback control at the level of each aggregator and distributed forecast-based optimisation at each associated user. The real-time operation is illustrated in Fig. 2, where it is assumed for simplicity purposes that each user represents only one flexible process, e.g. a battery system. However, under the proposed framework it is possible to address various flexible processes of different types (e.g. flexible loads).

The HEMS of the $j$ th user utilises an internal model for the optimisation of its flexible resource(s), over a time horizon of discrete steps $i$ starting at current discrete time control period $k$. Each aggregator defines in real-time a processed set-point trajectory ${ }^{4}$ $s_{a}(k+i \mid k)$, and then broadcasts this signal to all associated users (under a certain control area) at current time $k$. Subsequently, each user can decide internally, and in a voluntary manner how to respond to this control signal. The trajectory $s_{a}(k+i \mid k)$ actually represents the short-term objectives for the dispatch of resources within the portfolio of aggregator $a$, and is constructed based on

\footnotetext{
4 The notation $f(k+i \mid k)$ indicates that the function $f$ depends on the conditions at current time instant $k$.
}

information about: (close to) real-time requests from system operators for the provision of ancillary services, short-term predictions of supply and demand, and past control errors. Past control errors refer to deviations from the defined energy contract, whereas the requests from system operators refer to the TSO signals for loadfrequency control (LFC) and/or the DSO signals for peak shaving. The processed set-point trajectory $s_{a}(k+i \mid k)$ at current control period $k$ is a power trajectory (in Watt), and it consists of the sum of three trajectories: a short-term prediction trajectory of the power deviation values $\Delta P_{T S O, a}^{\text {prd }}(k+i \mid k)$ which accounts for requests from the TSO, a short-term prediction trajectory of the power deviation values $\Delta P_{D S O, a}^{p r d}(k+i \mid k)$ which accounts for requests from the DSO, and a power deviation trajectory $\Delta P_{a}^{\text {prd }}(k+i \mid k)$ which accounts for corrective actions in the case of deviations from the defined energy contract $E_{a}^{e c}(l)$ :

$$
\begin{aligned}
s_{a}(k+i \mid k)= & \Delta P_{T S O, a}^{p r d}(k+i \mid k)+\Delta P_{D S O, a}^{p r d}(k+i \mid k) \\
& +\Delta P_{a}^{p r d}(k+i \mid k)
\end{aligned}
$$

\subsection{Capacity management mode}

The main functionality of the DSO agent is to provide the proper control signals for maintaining the net demand and generation within the limits of network assets such as transformers and cables. Traditionally, peak demand could be predicted rather accurately based on historical data and weather forecasts, however, with the expectations for an ever increasing participation of DR resources, peak shaving activities in the future will have to be reconsidered, since the demand levels at a certain time will be subject not only to user behaviour and seasonal or weather conditions, but also to many other factors such as economic incentives, automated actions and external requests. For simplicity, let us assume that the DSO agent's goal is to maintain the power demand within the capacity limits of the MV/LV transformer, whereas in a more detailed scheme this concept could also be extended to other assets such as feeder lines.

At the MV/LV transformer level, the DSO agent is continuously informed (at each time step) by the aggregator(s) about the aggregate predicted power profile of associated users over a time horizon, and this profile has to be maintained within the power limits of the MV/LV transformer, based on Eq. (3):

$\left|P_{\text {grid }}^{\text {prd }}(k+i \mid k)\right|<P_{\max }^{\text {trans }}$

$P_{\text {grid }}^{\text {prd }}(k+i \mid k)=\sum_{a=1}^{A} \sum_{j \in a} P_{j}^{p r d}(k+i \mid k)$

where $P_{j}^{\text {prd }}(k+i \mid k)$ represents the predicted power profile of the $j$ th user and is based on Eq. (1). The short-term prediction trajectory of the power deviation values which accounts for requests from the DSO, can be defined as follows:

$$
\begin{aligned}
& \Delta P_{D S O}^{\text {prd }}(k+i \mid k) \\
& \quad=\left\{\begin{array}{cl}
P_{\max }^{\text {trans }}-P_{\text {grid }}^{\text {prd }}(k+i \mid k) & \text { if } P_{\text {grid }}^{\text {prd }}(k+i \mid k)>P_{\max }^{\text {trans }} \\
0 & \text { if } P_{\text {grid }}^{\text {prd }}(k+i \mid k) \leq P_{\max }^{\text {trans }}
\end{array}\right.
\end{aligned}
$$

Eq. (16) reflects the peak shaving requirements for the MV/LV transformer of the case study. In a real-life application, a DSO can decide which aggregator(s) to select for the provision of peak shaving services based on a merit order of peak shaving bids through a local single buyer market. For simulation purposes, in this paper, the peak shaving bids of both aggregators were equally treated, i.e. there were no prices attributed to the capacity bids for 
peak shaving services. Given that forecasted profiles and schedules might change considerably as time proceeds, the deviation values $\Delta P_{D S O}^{p r d}(k+i \mid k)$ are defined either close to real-time (i.e. just before the beginning of the $l$ th settlement period, and cover only that period), or in real-time within the current settlement period $q$. By letting the DSO to request peak shaving services, this creates a situation with natural incentives for the DSO to upgrade an aged network where peak shaving becomes necessary more frequently (and subsequently more expensive).

Distributed energy resources have the potential to provide ancillary services to grid operators (both at the transmission and distribution level), possibly with competing objectives. With the development of active distribution grid management, it becomes essential to establish procedures to identify and solve possible TSO/DSO conflicts that take place when requesting and/or procuring ancillary services from flexible distributed resources [22]. In the case that the investigated LV grid is expected to experience peak demand (or generation) and potential overloading of the MV/LV transformer, the DSO will request peak shaving actions which might be in contrast with requests by the TSO for downwards (or upwards) regulation. The following conditions can be distinguished $^{5}$ :

i. If an overloading of the MV/LV transformer is expected, i.e. $P_{\text {grid }}^{\text {prd }}(k+i \mid k)>P_{\max }^{\text {trans }}$ for $k+i \in q$, then the system enters the capacity management mode, i.e. the DSO requests peak shaving actions (the DSO signal is determined by using Eq. (16), whereas the bids for operating reserves that are in the opposite direction of the peak shaving objectives (i.e. reserves for downward regulation) shall be cancelled for the period of peak shaving ${ }^{6}$ (i.e. the TSO is informed that the capabilities originating from the respective part of the grid are limited due to peak shaving actions). The DSO may request part of the available flexibility for peak shaving, whereas the remaining flexibility capabilities are available for other purposes (e.g. TSO requests for upward regulation).

ii. If $P_{\text {grid }}^{\text {prd }}(k+i \mid k) \leq P_{\max }^{\text {trans }}$ but an overloading of the MV/LV transformer is expected in the case that the TSO requests operating reserves for downward regulation (equivalent to demand increase), i.e. $P_{\text {grid }}^{\text {prd }}(k+i \mid k)+P_{\text {bid }}^{u p}(q)>P_{\max }^{\text {trans }}$, then the system enters the optional capacity management mode, and the part of the bids for operating reserves for which $P_{b i d}^{u p}(k+i \mid k)>P_{\max }^{\text {trans }}-P_{\text {grid }}^{\text {prd }}(k+i \mid k)$ are cancelled. In the case of many active aggregators in the given part of the grid, the cancellation of bids for operating reserves can occur in an analogously manner among the active aggregators.

\subsection{Deviations from contracted energy}

The power deviation trajectory $\Delta P_{a}^{p r d}(k+i \mid k)$ is defined by each aggregator in real-time based on an energy balance equation for each settlement period that incorporates its defined energy contract, and forecasts over the lth settlement period. Let us assume, that we are at current time $k$, just before the beginning of the $l$ th settlement period. The power deviation trajectory, for $i=1, \ldots, n$, can be calculated as follows:

$\Delta P_{a}^{p r d}(k+i \mid k)=\Delta P_{a}^{d e v}(k)+\left(P_{a}^{e c}(l)-\sum_{j \in a} P_{j}^{p r d}(k+i \mid k)\right)$

\footnotetext{
5 Given that the investigated case study concerns a residential district, in this work, the focus is on peak demand conditions, and the case of network overloading due to peak generation is left out of the research scope.

6 In the case that the local aggregator is associated with a master aggregator at a higher level, then the cancelled bids for operating reserves may be directed to another part of the grid but for simplicity this is left out of the scope of the paper.
}

where the power deviation parameter $\Delta P_{a}^{\operatorname{dev}}(k)$ employed in Eq. (17) is defined by using Eq. (18). At the beginning of the current settlement period $q$, i.e. $k=15 \cdot(q-1)$, the power deviation parameter is zero. Subsequently, as time proceeds and current time $k$ is within the $q$ th settlement period, the first measured values of individual users become available. Starting at the beginning of the $q$ th settlement period and up to current time $k$, the difference between the submitted energy contract $E_{a}^{e c}(q)$ and the delivered energy (sum of the aggregate output but excluding the energy content due to requests by the system operators) define the delta energy content $\Delta E_{a}^{\operatorname{dev}}(\mathrm{k})=\Delta P_{a}^{\text {dev }}(\mathrm{k}) \cdot\left(q \cdot \tau_{s}-k \cdot \tau\right)$ that must be delivered until the end of the $q$ th settlement period for restoring the internal balance of aggregator $a$ :

$$
\begin{aligned}
& \text { if } k=15 \cdot(q-1) \text { then } \Delta P_{a}^{\operatorname{dev}}(k)=0 \\
& \text { else } \Delta P_{a}^{\operatorname{dev}}(k)=\frac{1}{\left(q \cdot \tau_{s}-k \cdot \tau\right)} \cdot \sum_{t=15 \cdot(q-1)+1}^{k}\left(P_{a}^{e c}(q)\right. \\
& \\
& \left.-\sum_{j \in a} P_{j}^{m s r}(t)+\Delta P_{T S O, a}(t)+\Delta P_{D S O, a}(t)\right) \cdot \tau
\end{aligned}
$$

where $P_{j}^{m s r}(t)$ is the net measured power supply and demand of the $j$ th user at time $t$.

\subsection{Operations of aggregators}

Each aggregator $a$ defines and broadcasts in real-time the processed set-point trajectory $s_{a}(k+i \mid k)$ to all associated users, $j \in a$. Each individual user obtains the trajectory $s_{a}(k+i \mid k)$, and checks whether it can partially contribute to the requested action by using a forecast-based optimisation approach (see the following sub-section). Then, that user sends back to the aggregator the individual reference trajectory signal $r_{j}(k+i \mid k)$ which includes the information about the part that can be fulfilled, i.e. the optimised schedule of its flexible resource(s). The aggregator receives the signal $r_{j}(k+i \mid k)$ from the $j$ th user and performs consistency checks to avoid an overshooting:

$\sum_{j \in a} r_{j}(k+i \mid k) \leq s_{a}(k+i \mid k)$

Then, the aggregator sends back to the user a discrete binary signal, i.e. $w_{j}(k) \in\{0,1\}$, to indicate rejection or acceptance with respect to $r_{j}(k+i \mid k)$, and the $j$ th user registers the optimised input(s), ${ }^{7} P_{j}^{\text {flex }}(k+i)$, to be applied at time $k+1$. Subsequently, the aggregator subtracts the scheduled (optimised) actions, recalculates and forwards the updated processed set-point trajectory and the whole process is repeated. The measured aggregate net supply and demand corresponding to aggregator $a$ at current time instant $k$ is defined as follows:

$P_{a}^{m s r}(k)=\sum_{j \in a}\left\{P_{j}^{\text {base, } m s r}(k)+P_{j}^{\text {flex,msr }}(k)\right\}$

The measured aggregate base load is summed up with the measured power demand of all flexible resources at the aggregator level (see Fig. 2). The aggregator's goal is to steer its associated users' aggregate power output according to market contracts (cleared market volumes) and the real-time requests from system operators.

\footnotetext{
7 For example, in the case that the flexible resource of the $j$ th user is a battery system, the optimised inputs correspond to the battery charging and discharging power schedule, i.e. $P_{j}^{\text {flex }}(k+i)=\left\{P_{d i s, j}^{b a t}(k+i), P_{c h, j}^{\text {bat }}(k+i)\right\}$.
} 


\subsection{The optimisation approach for system users}

Once the aggregator has broadcasted the set-point trajectory $s_{a}(k+i \mid k)$, then each associated user can respond in a voluntary fashion. The $j$ th user performs a forecast-based optimisation over the control horizon and sends back to the aggregator the reference trajectory signal about the part of the request that can be fulfilled. The reference trajectory signal of the $j$ th user is equal to the difference between the updated optimised power output, and the a priori optimised power output trajectory, over the optimisation horizon, and can be written as follows:

$r_{j}(k+i \mid k)=P_{j}^{p r d}(k+i \mid k)-P_{j}^{p r d}(k+i \mid t<k)$

The objective of the optimisation at each participating user is formulated as a function that either maximises the service provision offered to the aggregator (i.e. maximises the energetic contribution) while contributing to the coordination signal $s_{a}(k+i \mid k)$, or minimises the deviations from the a priori optimised output, e.g. in the case that the aggregator's portfolio is expected to be in balance. The goal is to optimise the flexible resources over a time horizon that spreads up to the end of the qth settlement period, starting at current time instant $k$ and subject to constraints:

$\# i=1, \ldots, n$. Note that $\max (n)=15$

\# $n$ is decreasing at each time step

if $s_{a}(k+i \mid k) \neq 0$

then $\max _{P_{j}^{f l e x}(k+i \mid k)} \sum_{i} r_{j}(k+i \mid k)$

for $k+i \in q$ while $r_{j}(k$
else $\min _{P_{j}^{f l e x}(k+i \mid k)} \sum_{i} r_{j}(k+i \mid k)$

where $P_{j}^{f l e x}(k+i \mid k)$ is the optimisation parameter which satisfies the objective function, i.e. the optimised input(s) of the flexible resource(s) of the $j$ th user.

\section{The financial settlement}

The financial settlement occurs after operations and allocates costs and benefits attributed to: (a) energy purchase and sell at the wholesale level, i.e. the DAM, (b) imbalances due to deviations from contracted energy, (c) revenues from the provision of aFRR to the TSO, and $(d)$ revenues from the provision of peak shaving services to the DSO. The annual costs/revenues in $€$ attributed to the DAM for aggregator $a$ are calculated as follows:

$C_{a}^{\text {market }}=\sum_{\text {day=1 }}^{365} \sum_{h=1}^{H} E^{e c}(h) \cdot \pi(h), \forall a$

where $\pi(h)$ is the cleared DAM price in $€ / M W h$ for the $h$ th hour. Any imbalance between scheduled and actual supply and/or demand is settled against the imbalance price for each aggregator $a$, and for each settlement period $l$ separately:

$E_{a}^{i m b}(l)=E_{a}^{e c}(l)-\sum_{j \in a} E_{j}^{m s r}(l)$

if $E_{a}^{i m b}(l)>0$

then $\pi_{a}^{i m b}(l)=\pi_{\text {sell }}^{i m b}(l)$

else $\pi_{a}^{i m b}(l)=\pi_{b u y}^{i m b}(l)$

$C_{a}^{i m b}=\sum_{\text {day=1 }}^{365} \sum_{l=1}^{L} E_{a}^{i m b}(l) \cdot \pi_{a}^{i m b}(l), \forall a$

where $C_{a}^{i m b}$ represents the annual imbalance cost in $€$ for aggregator $a$. Any energy imbalance during the $l$ th settlement period is settled against the imbalance price $\pi_{a}^{i m b}(l)$ an aggregator has to pay. This price is either the imbalance buy or sell price of the imbalance settlement system of the TSO. Any energy imbalance of aggregator $a$ that causes a surplus is settled against the imbalance sell price $\pi_{\text {sell }}^{i m b}(l)$, whereas a shortage is settled against the imbalance buy price $\pi_{b u y}^{i m b}(l)$. An aggregator and its associated users have potentially provided flexibility during real-time operations to solve system imbalances through aFRR provision, and/or congestion at the distribution level through the provision of peak shaving services. The financial settlement for the provision of ancillary services is applied separately for upwards and downwards power. The annual revenues of the ath aggregator for the provision of upwards and downwards regulation are calculated by using the following formulas:

$$
\begin{aligned}
& \Pi_{a}^{\text {reg,up }}=\sum_{\text {day }=1}^{365} \sum_{l=1}^{L} E_{a}^{\text {reg,up }}(l) \cdot \pi_{u p}^{\text {reg }}(l), \forall a \\
& \Pi_{a}^{\text {reg,down }}=\sum_{\text {day }=1}^{365} \sum_{l=1}^{L} E_{a}^{\text {reg, down }}(l) \cdot \pi_{\text {down }}^{\text {reg }}(l), \forall a
\end{aligned}
$$

where $E_{a}^{\text {reg,up }}(l)$ and $E_{a}^{\text {reg,down }}(l)$ are the energy volumes for upwards and downwards regulation provided during the $l$ th settlement period, and are calculated based on the actual delta-signal setpoints sent by the TSO, i.e. $\sum_{t \in l} \Delta P_{T S O, a}^{p r d}(t)$, whereas $\pi_{u p}^{\text {reg }}(l)$ and $\pi_{\text {down }}^{\text {reg }}(l)$ are the prices for regulating power and are defined by the maximum bid for upwards regulation and the minimum bid for downwards regulation activated by the TSO during the lth settlement period [29]. The prices $\pi_{u p}^{\text {peak }}(l)$ and $\pi_{\text {down }}^{\text {peak }}(l)$ for peak shaving requested by the DSO have been associated to the maximum bid for upwards regulation and the minimum bid for downwards regulation for aFRR activated by the TSO during any given day. The annual revenues of the ath aggregator for the provision of upwards and downwards power for peak shaving are calculated by using the following formulas:

$$
\begin{aligned}
& \Pi_{a}^{\text {peak,up }}=\sum_{\text {day }=1}^{365} \sum_{l=1}^{96} E_{a}^{\text {peak,up }}(l) \cdot \pi_{u p}^{\text {peak }}(l), \forall a \\
& \Pi_{a}^{\text {peak,down }}=\sum_{\text {day }=1}^{365} \sum_{l=1}^{96} E_{a}^{\text {peak,down }}(l) \cdot \pi_{\text {down }}^{\text {peak }}(l), \forall a
\end{aligned}
$$

where $E_{a}^{\text {peak,up }}(l)$ and $E_{a}^{\text {peak,down }}(l)$ are the energy volumes for upwards and downwards peak shaving services provided during the lth settlement period, and are calculated based on the actual deltasignal, sent by the DSO, i.e. $\sum_{t \in l} \Delta P_{D S O, a}^{p r d}(t)$.

\section{The case study}

The case study consists of a LV grid with $N=50$ connected residential users. There are two aggregators operating in the investigated LV grid, i.e. $A=2$, and the $N$ users are equally divided among them, i.e. users $j=1, \ldots, N / 2$ belong to the portfolio of aggregator $(a=1)$, whereas users $j=N / 2+1, \ldots, N$, belong to the portfolio of aggregator $(a=2)$. Each user agent represents a building which is equipped with local photovoltaic (PV) generation and an Energy Storage System (ESS). In Fig. 3, a schematic of the investigated case study, is provided. In the underlying business model, each user is associated with a single aggregator that acts as the link between that user and the system operators. ${ }^{8}$ The

\footnotetext{
8 Note that for simplicity reasons, only two aggregator companies are considered active in the investigated case study, whereas in real-life environments it is expected that many aggregators will be active in any given part of the grid. Nevertheless, the proposed framework is valid for the case of a competitive environment with many aggregators.
} 


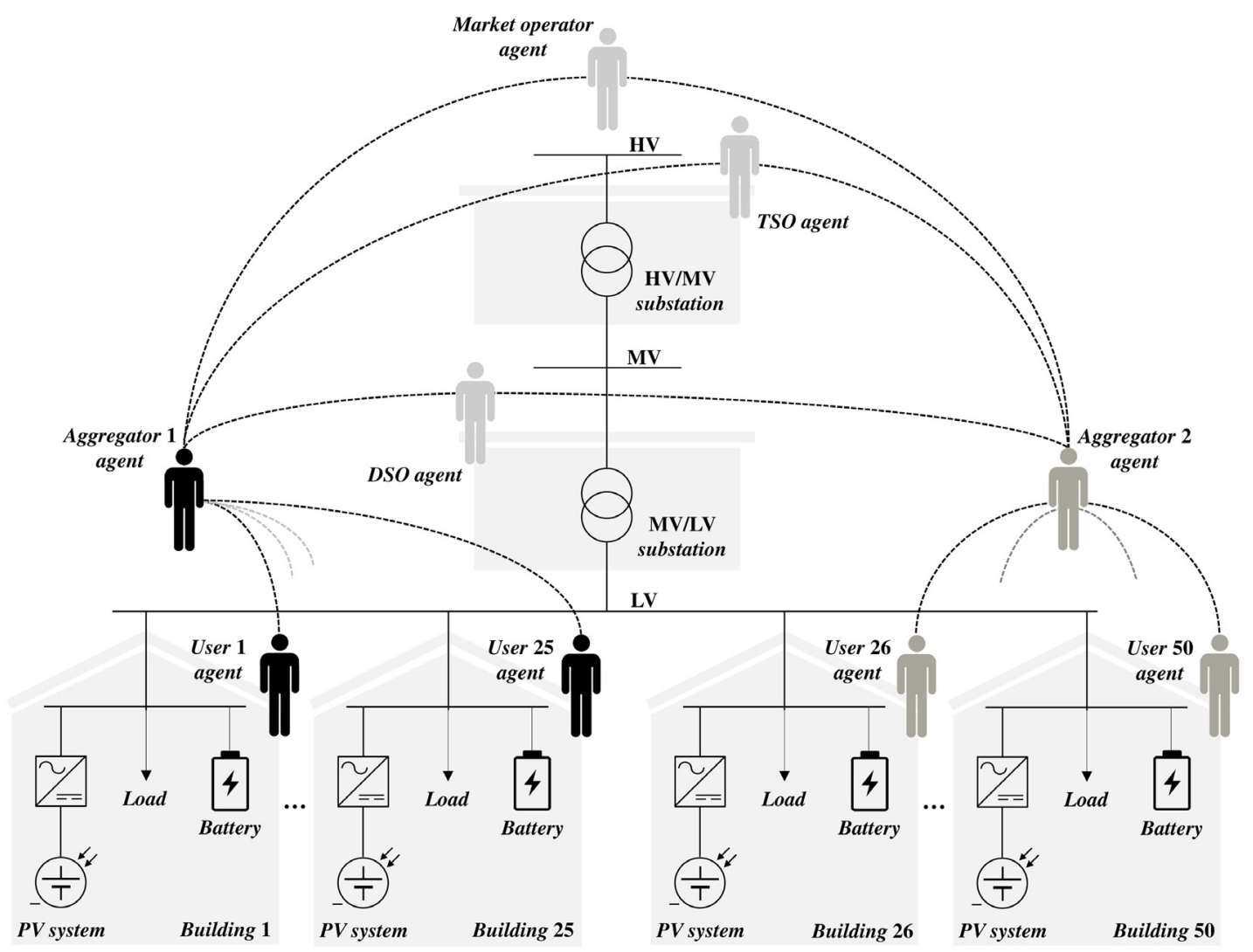

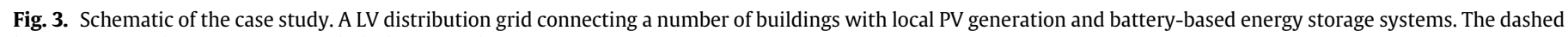
lines represent the communication links between the main actors.

aggregators of the case study are assumed to carry the role of the Balance Responsible Party (BRP), and the role of the supplier, but can also provide ancillary services to the grid operators. The case study does not only provide the basis for an economic assessment of an aggregator's potential in the investigated markets, but also contributes in clarity on how the proposed framework can be applied by providing in a clear and transparent manner all the mathematical formulas, models and assumptions.

For this case study, a load profile and a PV generation profile are assigned to each household, which form the non-controllable part of the net supply and demand of that household. The data are obtained from 50 different connections ( 25 profiles for residential demand, and 25 profiles for PV generation) and are characterised by a measurement interval of $15 \mathrm{~min}$. for the demand figures, and a minute interval for PV generation. A year of residential demand smart-meter data has been obtained from August 3, 2015 and a year of PV data has been obtained from January 1, 2015. The demand profiles were provided by a Dutch supplier and consist of metered data of anonymous consumers. The obtained PV generation data has been remapped, by time shifting the time series data, to match the seasonal pattern of the demand data, starting from August 3rd. For comparison reasons, the non-controllable load of aggregator 1 and 2 are identical. The PV generation data were collected from distributed PV systems, in the area of Utrecht and more information can be found in [30,31]. The total annual demand and PV generation of the 25 households in the case study correspond to $62 \mathrm{MWh}$ and $35 \mathrm{MWh}$ respectively. The months June and July are characterised by back-delivery to the grid, meaning that more electricity is produced than consumed. The maximum peak of the 50 households on the MV/LV transformer was $53 \mathrm{~kW}$ for back-delivery and $47 \mathrm{~kW}$ for delivery (demand). An overview of the monthly non-controllable energy is depicted in Fig. 4.

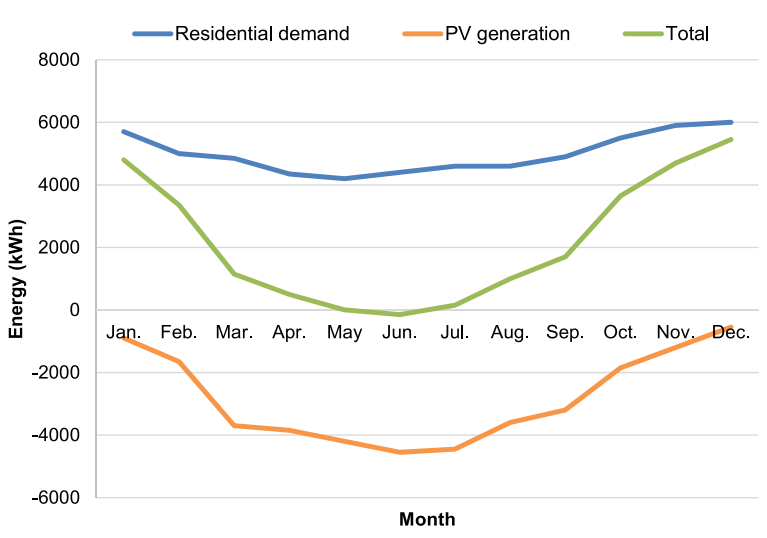

Fig. 4. The non-controllable energy figures of the case study.

Typical residential users are defined as having a connection with an annual average consumption of $3500 \mathrm{kWh}$ connected to the LV grid and a contracted capacity of $6 \mathrm{~kW}$ [32]. An assumption was made regarding the contracted capacity per connection corresponding to $2.5 \mathrm{~kW}$ per household. The ESS properties correspond to those of the Powerwall 2 battery system [33].

\subsection{The electricity market framework}

Recent developments in distribution grids, environmental policy, and the energy market liberalisation process, have opened up several opportunities for the provision of market-based products and services through aggregators [34]. In this context, the research focus on optimal ESS control has been shifted from the central role of the utility operator towards developing control strategies and 
applications for the provision of multiple services across different actors and markets $[24,25]$. In this paper, the focus is on three different applications, namely, energy arbitrage in the DAM of the European Power Exchange (EPEX) ${ }^{9}$ Netherlands [35], the provision of automatic Frequency Restoration Reserves (aFRR) to the Dutch TSO [29], and the provision of peak shaving services to the regional DSO.

In this section, we provide the time requirements of the investigated markets, including a description of our approach in the formulation of bids. Several scholars have addressed the formulation of bids for energy arbitrage and operating reserves through a joint optimisation approach. In [36], a two-stage stochastic optimisation model is proposed to define energy and tertiary reserve bids, whereas in [37] a stochastic mixed integer linear program is utilised for participation of a community energy system in both energy and reserve markets, while considering of local constraints. Contrary, our approach follows a sequential optimisation, where first the bids for the DAM are formulated by the Gate Closure Time (GCT), whereas bids for ancillary services are formulated close-toreal-time. This design choice is mainly due to the different timing and settlement periods of the investigated markets. The GCT of the DAM is at 12:00 on the day-ahead, i.e. the bids for the DAM must be submitted by the GCT and covering a forecasted period ranging at least from 12 to $36 \mathrm{~h}$ ahead. The wholesale trade of electricity is often complemented by markets for ancillary services, such as the aFRR market which has a lead time (i.e. the period between bidding and activation of a bid) of one full clock hour [34]. Ancillary services markets operate on a short time horizon, because the necessity of such services is often unknown until real-time operations. Thus, optimising jointly energy and reserve bids in the day-ahead planning phase would compromise the estimation accuracy of the available capacity for aFRR and peak shaving, especially considering an aggregation of DSM resources where the available flexible capacity is relatively erratic and it is essential to continuously maintain a forecast of resource availability. In the case that the flexible capacity is almost depleted, e.g. due to long and large request for frequency regulation, then it is important for the aggregator to submit updated bids for operating reserves to the grid operators. Furthermore, in the DAM, market members can trade hourly instruments, whereas the imbalance settlement system is based on ISPs of $15 \mathrm{~min}$. For these reasons, it has been decided as a design choice for our proposed framework to optimise energy and reserve bids in a sequential manner. The difference in the strategy of the two aggregators is that the first one is also utilizing the flexibility of its associated users for optimisation on EPEX DAM, whereas both aggregators are offering flexibility to the grid operators, i.e. provision of aFRR to the TSO and local congestion management services to the DSO.

\subsection{Assumptions}

The aggregators of the case study contribute to the provision of non-contracted aFRR to the TSO. Currently, this ancillary service requires the submission of bids with a min. size of $4 \mathrm{MW}$, whereas the activation minimum step is set to $1 \mathrm{MW}$. Due to the limited size of the aggregators, these requirements are disregarded. These requirements are due to a system that is relying also on manual human actions, but in the future we can assume that fully automated systems will be able to handle bids of any size. It is also assumed that a limited time span is required for updating the bids, which can be done until the beginning of an hour. A battery model was used to simulate the available flexibility within a household (see Section 6.3.). In practice, a household could also provide flexibility through demand-side response. A forecast of the non-controllable

\footnotetext{
9 Formerly Amsterdam Power eXchange (APX) Power NL.
}

load of each household was synthesised by adding a randomised error at the measured PV generation and demand profiles (see Section 6.4.).

For the reference scenario, the end-user (retail) electricity price is considered. The focus is on the energy component, which effectively represents the possibilities for end-user cost reduction that is accomplished by switching to another supplier. In theory there should be a relationship between end-user and wholesale electricity prices, however, in practice, there is low correlation between wholesale and retail prices [38]. Therefore, the research focuses on the margin for end-user cost reduction between the day-ahead wholesale and the retail price. The end-user electricity price (including taxes) in March 2017 in the Netherlands was $16.73 \mathrm{c} € / \mathrm{kWh}$, whereas the price breakdown corresponded to $37 \%$ for the energy component (including retail margins), 35\% for distribution, $10 \%$ for energy taxes and $17 \%$ for VAT [38]. Thus, the energy (electricity) component in the Netherlands in March, 2017 corresponded to $6.19 \mathrm{c} € / \mathrm{kWh}$, which was considered in the reference scenario, resulting into an aggregate energy invoice of $€ 3411.72$ for 25 households. For wholesale electricity trade the EPEX DAM clearing prices from 2016 were utilised [35], and for representing the TSO requests towards the aggregator (i.e. the LFC signal), the imbalance prices and volumes of the Dutch imbalance settlement system from 2016 were employed [39].

An assumption had to be made regarding the prices of peak shaving service requested by the DSO since this is an envisioned service which has not become operational yet. Based on the logic that local problems should be prioritised over issues at system level, prices for peak shaving should be at least equal to (or more profitable) than regulating power prices in order to provide a natural incentive to market participants to reserve flexibility for such purposes. Therefore, the prices for peak shaving have been associated to the maximum bid for upwards regulation and the minimum bid for downwards regulation activated by the TSO during any given day. Finally, a capacity constraint was assumed for the MV/LV transformer depending on the scenario.

\subsection{Battery model and constraints}

Each user is equipped by an ESS that is controlled by the HEMS. The ESS is a battery-based system and its state is characterised by its State of Energy $(S o E)$. The SoE of the $j$ th user at time instant $t$ is typically expressed as:

$$
\begin{aligned}
\operatorname{SoE}_{j}(t)= & \operatorname{SoE}_{j}(t-1)-\frac{1}{E_{n o m, j}} \cdot\left(\frac{1}{\eta_{d i s, j}} \cdot P_{d i s, j}^{b a t}(t)-\eta_{c h, j} \cdot P_{c h, j}^{b a t}(t)\right) \\
& \cdot \Delta(t), \forall t
\end{aligned}
$$

where $P_{c h, j}^{b a t}(t)$ and $P_{d i s, j}^{b a t}(t)$ are the charging and discharging power rates of the battery-based ESS of the $j$ th user, $\eta_{c h, j}$ and $\eta_{d i s, j}$ are the charge and discharge efficiency, respectively, and $E_{n o m, j}$ is the battery nominal capacity. The $S o E$ of the battery of the $j$ th user is bounded as follows:

$\operatorname{SoE}_{j, \min } \leq \operatorname{SoE}_{j}(t) \leq \mathrm{SoE}_{j, \text { max }}, \forall t$

where $S o E_{j, \min }$ and $S o E_{j, \max }$ are the minimum and maximum $S o E$ allowed, respectively.

For the $j$ th user of our case study, the power exchange with the grid based on Eq. (1) consists of the non-controllable part of the net supply and demand which includes non-controllable loads $P_{j}^{\text {load }}(t)$ and PV generation $P_{j}^{P V}(t)$, and the flexible part $P_{j}^{\text {flex }}(t)$ which is attributed to the $j$ th EES which is controlled by a HEMS.

$P_{j}^{\text {base }}(t)=P_{j}^{\text {load }}(t)-P_{j}^{P V}(t), \forall t$

$P_{j}^{f l e x}(t)=P_{c h, j}^{b a t}(t)-P_{d i s, j}^{b a t}(t), \forall t$ 
Eqs. (32)-(33) are used for determining the energy volumes to be traded at the day-ahead market, based on Eq. (4), and also become part of the power constraint regarding the power exchange with the grid in the day-ahead optimisation problem (see Eq. (6)). The constraints regarding the charging/discharging power of the $j$ th EES can be written as follows:

$0 \leq P_{c h, j}^{b a t}(t) \leq P_{c h, j, \max }^{b a t}, \forall t$

$0 \leq P_{\text {dis }, j}^{b a t}(t) \leq P_{d i s, j, \max }^{b a t}, \forall t$

where $P_{d i s, j, \max }^{b a t}$ and $P_{c h, j, \text { max }}^{\text {bat }}$ are constant values defined as power boundaries for each $j$ th user. All the residential users are connected to the same MV/LV transformer. The DSO might procure peak shaving services from distributed resources, through the aggregators, to maintain the power exchange with the MV grid within the transformer capacity limits (see Eq. (3)).

During the operational planning phase, the day-ahead optimisation problem is subject to additional constraints, i.e. the power charge and discharge of the battery described in Eqs. (34)-(35), and the following constraint which ensures that the $S o E$ at the end of the day is at the same level as at the beginning of the day:

$\operatorname{SoE}_{j}^{\text {das }}(H)-\operatorname{SoE}_{j}^{\text {das }}(0)=0, \forall j$

where $\operatorname{SoE}_{j}^{\text {das }}(0)$ and $\operatorname{SoE}_{j}^{\text {das }}(H)$ represent the scheduled $\operatorname{SoE}$ at the beginning of the day (i.e. 00:00) and at the end of the day (i.e. 24:00) respectively. An example of the optimised schedule of the aggregate EES for the first aggregator $(a=1)$ is illustrated in Fig. 5 together with the market prices. Furthermore, next to calculating the available capacity per user connection (see Eqs. (7)(8)) for determining the energy capabilities for the provision of ancillary services, it is necessary to do this also for each ESS:

$P_{j}^{\text {bat }, u p}(h)=P_{d i s, j, \text { max }}^{\text {das }, \text { at }}-P_{j}^{\text {das,flex }}(h)$

$P_{j}^{\text {bat,down }}(h)=P_{c h, j, \max }^{\text {das, } a t}-P_{j}^{\text {das,flex }}(h)$

where $P_{j}^{\text {bat,up }}(h)$ and $P_{j}^{\text {bat,down }}(h)$ represent the available capacity of the battery system of the $j$ th user for up- and down-regulation. $P_{c h, j, \text { max }}^{\text {das, } a t}$ and $P_{\text {dis, }, j, \text { max }}^{\text {dax }}$ represent the max. allowed day-ahead charging and discharging power of the $j$ th battery system according to Eqs. (34)-(35). Part of the SoE of a battery system can be reserved for the provision of ancillary services in real-time. How much of a battery capacity to allocate for different purposes relies on the market strategy of an aggregator company. A decision that also relates to the technical characteristics of a battery system, the potential of a certain application as well as to the associated risk. For instance, the application of energy arbitrage in the day-ahead market appears to be highly susceptible to the efficiency of the storage system and the daily price development and volatility. On the contrary, contribution to system balancing appears to be a more profitable strategy. However, the latter application involves higher risks due to the stochastic nature of the imbalance market and the inherent difficulty to accurately predict the system state a priori, whereas contemporary day-ahead markets are characterised by a diurnal pattern of prices, which makes the forecasting of dayahead market prices more plausible [25]. For aggregator 1 of the case study, it was decided in an heuristic manner to allocate $40 \%$ of the capacity for day-ahead energy arbitrage, $40 \%$ for ancillary services, whereas $20 \%$ is reserved to preserve a long lifetime of the battery $(S o E>10 \%$ and $S o E<90 \%)$. The available power capacity for the battery of the $j$ th user for charging (down-regulation) and discharging (up-regulation) is determined as follows:

$P_{c h, j}^{\text {bat,down }}(h)=\frac{E_{n o m, j}}{\eta_{c h}} \cdot \frac{\operatorname{SoE}_{j}(h-1)-S o E_{j, \text { min }}^{r t}}{\tau_{h}}$

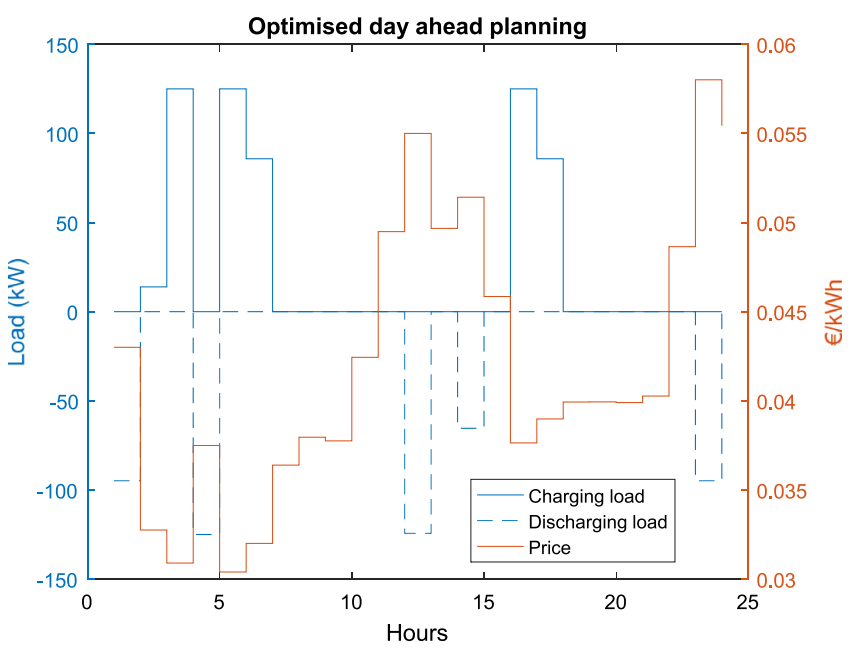

Fig. 5. Simulated aggregate profile of the optimised battery-based systems and market prices for the 25 residences that belong to the portfolio of the first aggregator for an example day.

$P_{d i s, j}^{\text {bat }, u p}(h)=\eta_{\text {dis }} E_{n o m, j} \cdot \frac{\operatorname{SoE}_{j, \max }^{r t}-\operatorname{SoE}_{j}(h-1)}{\tau_{h}}$

where $S o E_{j, \text { min }}^{r t}$ and $S o E_{j, \text { max }}^{r t}$ represent the minimum and maximum allowed $S o E$ in real-time for the $j$ th user. Subsequently, each $j$ th user can determine the available capacity for upwards and downwards regulation of its EES subject to technical constraints and commitments that are made a priori.:

$P_{j}^{\text {flex,up }}(h)=\min \left(P_{j}^{\text {bat }, u p}(h), P_{\text {dis, }, j}^{\text {bat } u p}(h)\right)$

$P_{j}^{\text {flex,down }}(h)=\min \left(P_{j}^{\text {bat,down }}(h), P_{\text {ch,j }}^{\text {bat,down }}(h)\right)$

For the case of the $j$ th user, the energy capabilities for upwards $P_{j}^{u p}(h)$ and downwards $P_{j}^{\text {down }}(h)$ regulation at hour $h$ are determined, based on (11) and (12). An example of the aggregate energy capabilities of the first aggregator $(a=1)$ for the provision of operating reserves is provided in Fig. 6, both for upwards and downwards regulation. Note that the plotted curves of energy capabilities for operating reserves in Fig. 6 are equal to the feedand take bid, meaning that the energy capabilities are directly submitted as a bid. An aggregator could dynamically change the bid prices, depending on the available energy capabilities, or the cost of provision of flexibility. However, for this case study the price bid strategy is kept simple by setting the EPEX day-ahead price for up- and down-regulation bids. The idea is that a system-user is only willing to adjust its consumption, when there is a financial incentive.

\subsection{Measured and forecasted data}

Measured data of local PV generation, and demand profiles of residential users were utilised as input for simulation purposes. For the day-ahead planning phase, the optimisation algorithm requires forecasted profiles of local demand and generation as input. For this purpose, forecasted profiles were synthesised, based on the actual measured data, by incorporating a random error in both the metered PV generation and demand data of the $j$ th user:

$P_{j}^{P V, p r d}(t)=P_{j}^{P V, m s r}(t) \cdot\left(1+\delta_{j}^{P V}(t)\right)$

$P_{j}^{\text {load,prd }}(t)=P_{j}^{\text {load,msr }}(t) \cdot\left(1+\delta_{j}^{\text {load }}(t)\right)$ 


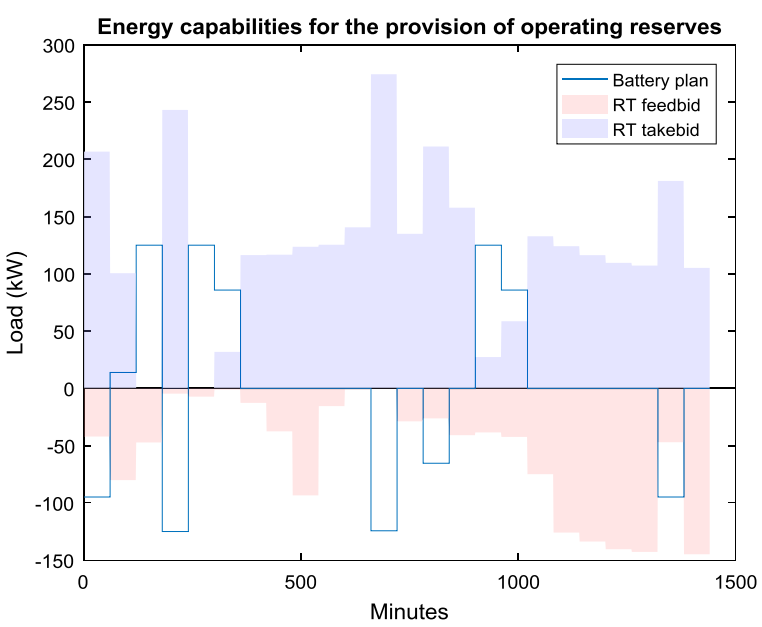

Fig. 6. Simulated aggregate bids for the provision of operating reserves compared to the charging and discharging day-ahead schedule of the battery-based energy storage systems which correspond to the 25 residences that belong to the portfolio of the first aggregator for an example day.

where $P_{j}^{P V, p r d}(t)$ and $P_{j}^{\text {load,prd }}(t)$ represent the forecasted data, $P_{j}^{P V, m s r}(t)$ and $P_{j}^{\text {load,msr }}(t)$ are the actual measured data, and $\delta_{j}^{P V}(t)$ and $\delta_{j}^{\text {load }}(t)$ represent the forecast errors of PV generation and demand respectively for the $j$ th user at time $t$. The errors consist of two components, i.e. a random general error and a random userspecific error:

$\delta_{j}^{P V}(t)=\delta^{P V, g e n}(t)+\delta_{j}^{P V, s p c}(t)$

$\delta_{j}^{\text {load }}(t)=\delta^{\text {load,gen }}(t)+\delta_{j}^{\text {load,spc }}(t)$

where the general error components $\delta^{P V \text {,gen }}$ and $\delta^{\text {load,gen }}(t)$ represent a day-ahead weather forecast error and the load dependency on weather, whereas the specific error components $\delta_{j}^{P V, s p c}(t)$ and $\delta_{j}^{\text {load,spc }}(t)$ correspond to PV generation which is attributed to local weather circumstances, e.g. local clouding conditions (as weather forecast spatial resolution usually is too course), and for the load it is attributed to variations in user behaviour, e.g. the use of appliances. A common way to express the accuracy of a forecasting error is by using the mean absolute percentage error (MAPE) measure which expresses accuracy as a percentage:

MAPE $=\frac{100}{n} \sum_{t=1}^{n}\left|\frac{A_{t}-F_{t}}{A_{t}}\right|$

where $n$ represents the number of samples, $A_{t}$ is the actual value and $F_{t}$ is the forecast value. Typically the day-ahead MAPE of forecasted aggregate consumption is in the range of $1.5 \%-3 \%$ [40], and it is dependent on the level of aggregation, the users' variation and weather conditions. The MAPE for forecasted PV power generation is typically larger due to its higher dependency on weather conditions. The authors in [41], obtained hourly day-ahead MAPE in the range of $10 \%-21 \%$ for each month of forecasted PV power generation by using three different neural network models. In a more recent study, the authors in [42], employed an artificial neural network based approach to predict the PV power output of a sunny day and a rainy day by using historical power data and weather data, and they obtained a MAPE of $10.06 \%$ for the sunny day, and a MAPE of $18.89 \%$ for the rainy day. The above mentioned references provide indicative MAPE values as a basis for developing the simulation scenarios in the following section. Within this case study a $12 \mathrm{~h}$ ahead forecast is required, for which the synthetic forecast profiles were created at a user level, then aggregated at each aggregator level. The general error component is expected to contribute to higher MAPE values, whereas many of the specific errors of individual users will cancel each other out at an aggregated level. The general and specific error components for the PV generation and demand in Eqs. (45)-(46) are determined as follows:

$$
\begin{aligned}
& \delta^{P V, \text { gen }}(t)=\alpha^{P V, \text { gen }}+\beta^{P V, \text { gen }} \cdot \operatorname{rand}(t) \\
& \delta_{j}^{P V, s p c}(t)=\alpha^{P V, s p c}+\beta^{P V, s p c} \cdot \operatorname{rand}_{j}(t) \\
& \delta^{\text {load,gen }}(t)=\alpha^{\text {load,gen }}+\beta^{\text {load,gen }} \cdot \operatorname{rand}(t) \\
& \delta_{j}^{\text {load,spc }}(t)=\alpha^{\text {load,spc }}+\beta^{\text {load }, s p c} \cdot \operatorname{rand}_{j}(t)
\end{aligned}
$$

where $\operatorname{rand}(t)$ and $\operatorname{rand}_{j}(t)$ return a single uniformly distributed random number in the interval $(0,1)$ for all users and each $j$ th user respectively, and factors $\alpha^{P V, \text { gen }}=-0.35, \beta^{P V, \text { gen }}=0.7$, $\alpha^{P V, \text { spc }}(t)=-0.2, \beta^{P V, s p c}=0.4, \alpha^{\text {load,gen }}=0.15, \beta^{\text {load,gen }}=$ $0.3, \alpha^{\text {load,spc }}=-0.2$, and $\beta^{\text {load,spc }}=-0.4$ were determined in a heuristic manner so that the MAPE values for the synthesised forecasted profiles for PV generation and demand correspond to those of the simulation scenarios (see Table 2).

\subsection{Scenarios}

Three scenarios were developed for this study, which are differentiated on the basis of three main parameters, i.e. the levels of forecast error in PV generation and demand, and the MV/LV transformer capacity. The first and second scenario are characterised by a low and a medium forecast error, along with a congestion capacity constraint of $125 \mathrm{~kW}$ corresponding to the MV/LV transformer. ${ }^{10}$ In the third scenario a high forecasting error is applied while the capacity of the MV/LV transformer has been lowered to $90 \mathrm{~kW}$, i.e. congestion arises more frequently and volumes of congestion are larger. The first scenario corresponds to a low forecast error of 10.2\% MAPE for PV generation and 2.5\% MAPE for consumption and the second scenario to a high forecast error of $15.7 \%$ and $3.3 \%$ respectively. For simulating an extreme case, an even higher forecasting MAPE and a more stringent transformer capacity constraint was set for scenario three, where the MAPE for PV generation was set to $18.2 \%$, while the MAPE for consumption was more than doubled to $7.4 \%$. The specifications of the case study are listed in Table 1 , whereas an overview of the simulation scenarios is listed in Table 2.

\section{Simulations and results}

In order to analyse the response of the aggregate system, the data collection and the developed simulation scenarios focus on the Netherlands and the reference year 2016. ${ }^{11}$ All software codes and simulations were implemented in Matlab environment [43].

\footnotetext{
10 In practice, a typical nominal capacity of a MV/LV transformer in the Dutch grid is about $400 \mathrm{~kW}$, delivering power to $250-350$ households. For simulation purposes, the developed scenarios address transformer capacities of 90/125 kW corresponding to the case study of 50 households.

11 The data set used as input for the simulation model, also includes data for demand, PV generation and market figures from proximate years, i.e. 2015-2017, however, the impact on the results is negligible due to small annual variations, thus the data input is considered as credible for addressing the reference year 2016.
} 
Table 1

Specifications of the case study.

\begin{tabular}{ll}
\hline Description & Value \\
\hline Contracted capacity per connection & $6 \mathrm{~kW}$ \\
MV/LV transformer capacity (depending on the scenario) & $125 / 90 \mathrm{~kW}$ \\
Max. battery charge and discharge rate & $5 \mathrm{~kW}$ \\
Nominal battery capacity & $13.5 \mathrm{kWh}$ \\
Battery roundtrip efficiency & $90 \%$ \\
Maximum allowed SoE & $90 \%$ \\
Minimum allowed SoE & $10 \%$ \\
Minimum day-ahead allowed $S o E$ for aggregator 1 & $\mathrm{SoE}_{j}^{\text {das }}(0)-20 \%$ \\
Maximum day-ahead allowed $S o E$ for aggregator 1 & $\mathrm{SoE}_{j}^{\text {das }}(0)+20 \%$ \\
Minimum day-ahead allowed $S o E$ for aggregator 2 & $\mathrm{~N} / \mathrm{A}$ \\
Maximum day-ahead allowed $S o E$ for aggregator 2 & $\mathrm{~N} / \mathrm{A}$ \\
\hline
\end{tabular}

Table 2

Overview of the three simulation scenarios.

\begin{tabular}{llll}
\hline Scenario & 1 & 2 & 3 \\
\hline Transformer capacity (kW) & 125 & 125 & 90 \\
PV generation MAPE (\%) & 10.2 & 15.7 & 18.2 \\
Residential demand MAPE (\%) & 2.5 & 3.3 & 7.4 \\
\hline
\end{tabular}

\subsection{Overview of results}

The results were obtained for three different scenarios and for two different strategies of the two aggregators. The discrepancies in the scenarios and strategies have resulted in differences in the financial settlement for the two aggregators of the case study. The financial difference compared to the reference scenario ranges from $€ 11,063$ for scenario 2 for aggregator 2 , to $€ 14,504$ for scenario 3 and aggregator 1 , which reflect an annual gain of $€ 443-$ $€ 580$ per residential user. It should be taken into account that any costs associated with the provision of the flexibility, e.g. hardware, software and labour, are not included. A graphical overview of the cost and revenues for both the reference and three scenarios is displayed in Fig. 7, whereas the detailed figures are listed in Table 3. Overall, it was observed that the increase in forecasting error has a relatively small effect on the imbalance volume (and associated cost) that is settled among the three scenarios. An increase in forecast error leads to a slight decrease in profit made by an aggregator. The first aggregator has the most profitable strategy in each scenario, in which the aggregator optimises part of the flexible energy capabilities on the EPEX DAM. However, when comparing profit per unit of provided flexibility, aggregator 2 has the best strategy. The cost of peak-shaving service increases significantly when the constraint of the MV/LV transformer capacity is lowered in scenario 3, which creates an incentive for the DSO to enhance the grid capacity and avoid additional costs for procuring peak shaving services.

\subsection{Comparison of scenarios 1 and 2}

Aggregator 1 generated a profit of $€ 1768$ through energy arbitrage at the EPEX DAM. However, a lower profit was settled at $€ 827$ because of cost incurred by the non-controllable part of the load. The cost of the non-controllable load was $€ 941$, i.e. equal to the settlement of aggregator 2 . The difference between the day-ahead settlement of scenario 1 and 2 of aggregator 2 is purely random, attributed to the forecasting error.

In real-time operations, aggregator 1 has half of the available flexible capacity compared to aggregator 2 . However, aggregator 1 can provide more flexibility in the case that the requests of the DSO and/or TSO are in the opposite direction of the day-ahead charging and discharging schedules optimised for the EPEX DAM. This explains why aggregator 1 , which reserved only half of its flexibility for real-time operations, does not receive only half of the profits compared to aggregator 2 .
Within the model, congestion can occur due to the scheduled charging and discharging of the battery systems to address market optimisation (see Fig. 5). In that case, the scheduled position of aggregator 1 (i.e. cleared bids on the EPEX DAM) which result into congestion can be disregarded and flexibility can be provided to the DSO in the form of peak shaving services. For this reason, aggregator 1 receives more revenue from the DSO compared to aggregator 2. When comparing the revenues from regulating power (aFRR provision), aggregator 1 receives $77 \%$ of the revenues from regulating power compared to aggregator 2 . For the first scenario, the total revenues from ancillary services provision are $€ 6934$ for aggregator 1 and $€ 8690$ for aggregator 2 . The increased revenue of aggregator 2 during real time operations ( $€ 1755)$ compensates almost exactly for the difference due to energy arbitrage in DAM $(€ 1768)$. The approximate match in revenues between aggregator 1 and 2 is a coincidental result.

In line with expectations, scenario 1 leads to a higher profit compared to scenario 2. Aggregator 1 and aggregator 2 generate a profit in scenario 1 of $€ 7745$ and $€ 7678$ respectively. The profits generated by aggregator 1 and 2 in scenario 2 are $€ 34$ and $€ 27$ lower compared to scenario 1 , resulting in a profit of $€ 7710$ and $€ 7651$ respectively. Although a reduction of revenues was expected as a result of increased imbalance costs, bids for operating reserves were adjusted on an hourly basis, thereby preventing larger imbalances. Note that the bids for ancillary services within the model are constructed based on the aggregate flexible energy capabilities of individual users, which are dependent on several user constraints. Because of forecasting errors attributed to individual users, the users' connection capacity constraint can become a limiting factor in the formulation of a bid. However, when the forecast error is in the opposite direction, the bids are limited by the maximum charging and discharging rate of the battery. As a result, the constraints can become limiting factors when formulating a bid, resulting into smaller bids for ancillary services. For this reason, lower revenues are generated from ancillary services provision, but not necessarily leading to higher imbalance costs.

A noteworthy outcome is the higher imbalance cost of aggregator 2, compared to aggregator 1 . This is due to the larger bids for ancillary services of aggregator 2 , and the difficulty to fulfil those bids whenever requested by the TSO and/or DSO, compared to aggregator 1 who has reserved half of its flexible capacity for DAM optimisation. As a result, the imbalance volume that has to be settled for aggregator 2 is often higher.

Overall, aggregator 1 outperforms aggregator 2 in terms of net profits in all scenarios. However, when it comes to value created per unit of flexible energy provided., aggregator 1 has provided almost double the volume of flexible energy compared to aggregator 2. This is due to the strategy of aggregator 1 to optimise part of its flexible capacity for energy arbitrage at the EPEX DAM. The EPEX DAM has a lower volatility in prices with an hourly interval, while ancillary services are characterised by higher price volatility and shorter durations of the requests. In Fig. 8, a boxplot is plotted of the daily profits of the two aggregators for a whole year regarding scenario 2 . The strategy of aggregator 1 is characterised by a steady cash-flow generated through energy arbitrage at the EPEX DAM, whereas aggregator 2 has a much higher volatility in revenues generated through ancillary services markets.

In Figs. 9 and 10 the combined measured load of the two aggregators is shown for an example day of scenario 2 and is compared to the TSO request for regulating power and the DSO request for peak shaving respectively. Provided flexibility in real-time operations is visible by the difference between the day-ahead plan and the measured power. Overall, there is a high correlation between the requests for ancillary services and the provided flexibility. 


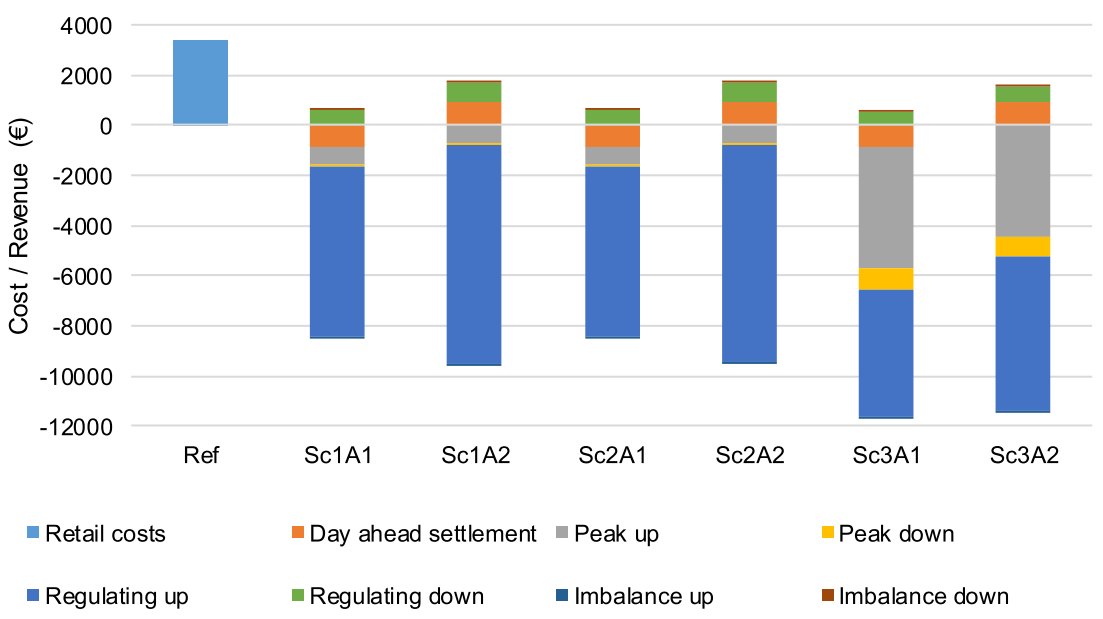

Fig. 7. Overview of costs and revenues for all scenarios (negative figures represent profits). The results are presented for each scenario and for each aggregator, e.g. Scenario 1 for Aggregator 1 (Sc1A1).

Table 3

Cost settlement overview for two aggregators in three different scenarios (negative monetary values indicate revenues).

\begin{tabular}{|c|c|c|c|c|c|c|}
\hline \multirow{2}{*}{$\begin{array}{l}\text { Scenario } \\
\text { Aggregator }\end{array}$} & \multicolumn{2}{|l|}{1} & \multicolumn{2}{|l|}{2} & \multicolumn{2}{|l|}{3} \\
\hline & 1 & 2 & 1 & 2 & 1 & 2 \\
\hline Charge volume (MWh) & 252 & 131 & 252 & 131 & 237 & 114 \\
\hline Discharge volume (MWh) & 227 & 118 & 227 & 118 & 213 & 103 \\
\hline MAPE PV generation (\%) & 10.2 & 10.2 & 15.7 & 15.7 & 18.2 & 18.2 \\
\hline MAPE demand (\%) & 2.5 & 2.5 & 3.3 & 3.3 & 7.4 & 7.4 \\
\hline $\mathrm{MV} / \mathrm{LV}$ transformer $(\mathrm{kW})$ & 125 & 125 & 125 & 125 & 90 & 90 \\
\hline Day-ahead settlement $(€)$ & -827.22 & 941.46 & -823.91 & 944.79 & -828.57 & 940.10 \\
\hline Peak up $(€)$ & -725.67 & -683.72 & -733.74 & -692.15 & -4893.23 & -4430.82 \\
\hline Peak down $(€)$ & -98.33 & -105.83 & -96.54 & -107.07 & -813.94 & -808.46 \\
\hline Regulating up $(€)$ & -6759.80 & -8679.50 & -6737.67 & -8655.64 & -5083.30 & -6136.04 \\
\hline Regulating down $(€)$ & 649.44 & 779.07 & 655.91 & 784.57 & 519.84 & 638.81 \\
\hline Imbalance up $(€)$ & -12.65 & -13.88 & -11.09 & -13.99 & -10.71 & -24.98 \\
\hline Imbalance down $(€)$ & 33.55 & 84.30 & 36.79 & 88.18 & 18.10 & 84.11 \\
\hline Yearly settlement $(€)$ & -7744.67 & -7678.10 & -7710.25 & -7651.31 & -11091.81 & -9737.27 \\
\hline
\end{tabular}

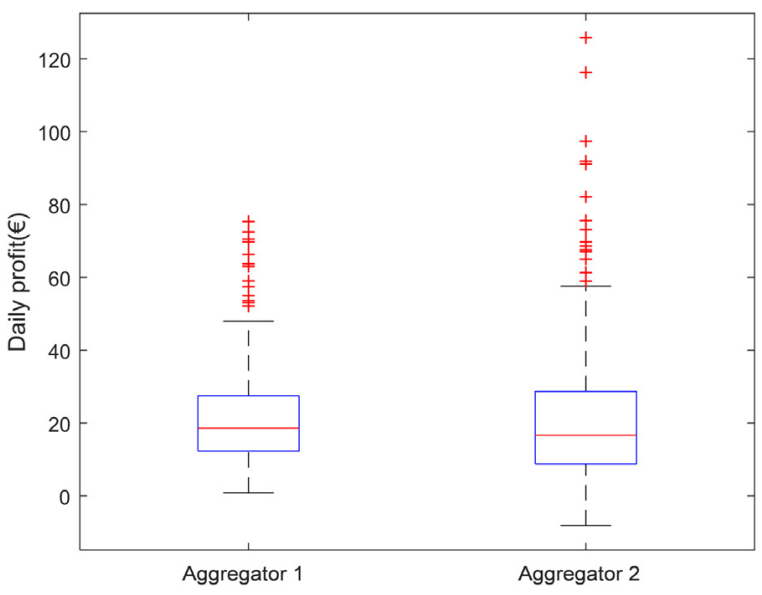

Fig. 8. Boxplot of the daily costs/benefits in scenario 2, for aggregator 1 and 2 .

\subsection{Scenario 3 compared to Scenarios 1 and 2}

A more extreme scenario is simulated in scenario 3, with a harder constraint regarding the MV/LV transformer capacity and a higher forecasting error. The MV/LV transformer capacity has been lowered from $125 \mathrm{~kW}$ to $90 \mathrm{~kW}$. During the day-ahead planning aggregator 1 and 2 obtain similar results as in scenarios 1 and 2, whereas any differences are purely random based on the forecasting errors. Aggregator 1 achieves a profit of $€ 828$ which includes

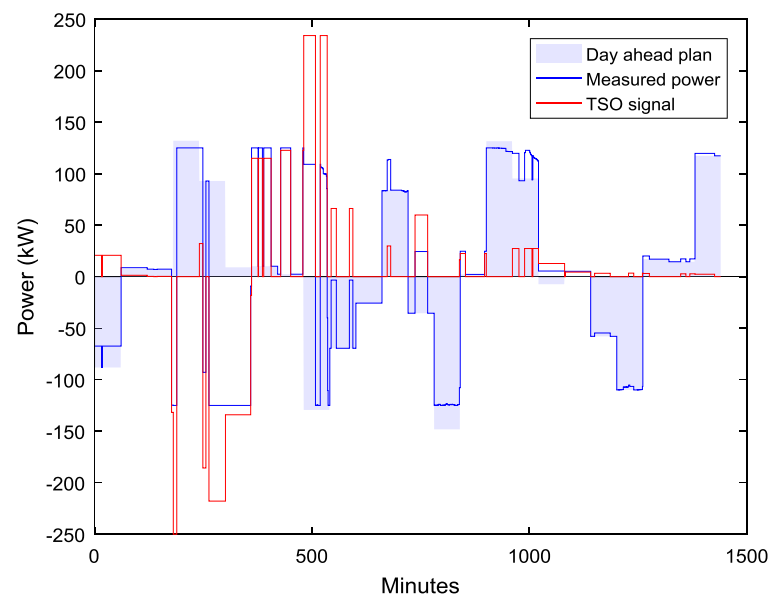

Fig. 9. TSO signal and system response (e.g. aggregate power profiles of one example day to illustrate the correlation of the aggregate demand with the dayahead schedules and the TSO signal).

the costs attributed to the non-controllable part of the load and the revenues attributed to energy arbitrage by optimising the flexible resources, whereas aggregator 2 faces a cost of $€ 940$ attributed to the non-controllable load. The requests by the DSO for peak shaving services have increased, along with the revenues for the aggregators. Aggregator 1 and 2 generated a revenue of $€ 5707$ and $€ 5239$ for peak shaving in scenario 3 , up from a profit of $€ 830$ and 


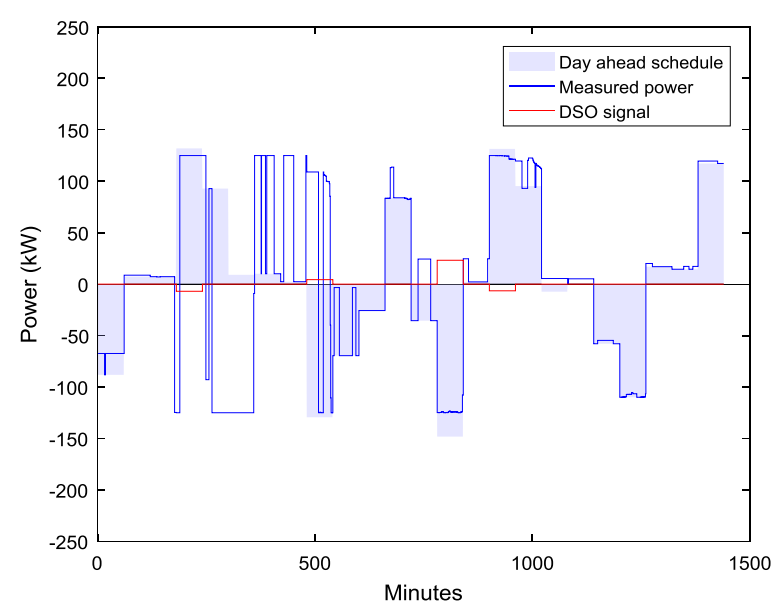

Fig. 10. Congestion management by the DSO (e.g. aggregate power profiles of one example day to illustrate the peak shaving mode).

$€ 799$ in scenario 2 . For the DSO this would lead to a significant increase of costs of more than $€ 100$ per user. It is expected that such an increase in costs for peak shaving will stimulate the DSO to invest into grid capacity enhancement.

The revenues from regulating power have decreased in scenario 3 due to the fact that the provision of peak shaving services are prioritised over the provision of regulating power, thus leaving less flexibility available for aFRR. The provision of regulating power in terms of profits in scenario 3 for aggregator 1 and 2 were $€ 4563$ and $€ 5497$ respectively, down from $€ 6082$ and $€ 7871$ in scenario 2 . The total provided flexibility in terms of energy volume, has decreased in scenario 3 due to an increase in the total time that the system is in capacity management mode.

\section{Discussion \& conclusions}

A hierarchical control framework is proposed that is characterised by decentralised decision making, distributed computation, and ensures that no sensitive data, related to user behaviour, requirements and schedules, are transmitted outside the premises of a user. All users' objectives and constraints are processed locally and only aggregate and reference data are communicated outside the users' premises. Therefore, the proposed control scheme can tackle privacy concerns and contribute to higher user acceptance. The proposed framework is generic and can be extended to include additional flexible resources, other than the battery-based EES which was exclusively utilised for validation purposes. The focus is on enabling demand-side resources, through aggregators, to participate in energy transactions in wholesale markets, as well as the provision of ancillary services to the grid both at the transmission and distribution levels.

A simulation model was developed and results were obtained for three different scenarios. The first and the second scenario focused on different levels of forecasting errors, whereas the third scenario focused on a harder LV/MV transformer capacity constraint to simulate an aged distribution grid. In each scenario, the participation of two aggregators with different strategies was simulated. The first aggregator utilised its flexible capabilities during both the operational planning for DAM optimisation, and in real-time for the provision of ancillary services, whereas the second aggregator only provided ancillary services. The outcome of computer simulations provided an informative insight into the differences between the strategies of the two aggregators, and the potential of the investigated markets by utilising historical market data. Results show a significant potential of offsetting electricity costs and generating additional revenues for residential customers equipped with photovoltaic systems and energy storage capacity. From the obtained results, it can be concluded that the first aggregator generates the highest profit, whereas the second aggregator has the highest profit per unit of provided flexible energy. This outcome is mainly attributed to the lower price volatility in the DAM which is based on hourly settlement periods, compared to a higher volatility in prices for ancillary services with a shorter settlement period. An increase in the forecast error, induced harder constraints in the formulation of bids for ancillary services, and resulted into smaller bids and a decrease in generated revenue. The increase in forecasting error had a relatively small effect on the imbalance cost that was settled among the three scenarios for both aggregators, demonstrating the ability of the algorithms to adapt the aggregate demand and/or supply in real-time. A decrease in the nominal capacity of the MV/LV transformer in the third scenario resulted into a large increase of revenues from peak shaving services, and a decrease of revenues from the provision of balancing reserves. The cost of peak shaving services to prevent grid congestion are paid by the DSO to the aggregators, thus a large increase in procurement costs for such services is expected to stimulate the DSO to invest into distribution grid capacity enhancement.

The proposed conceptual framework enables novel organisational and cooperative structures for the efficient energy management of demand-side resources in buildings, and supports the development of new products, services, business models and appropriate regulatory frameworks. Future work will address the emergency and outage system modes and incorporate additional flexible distributed resources (e.g. inverter-based PV systems and EVs with varying battery capacities) and power network models in the proposed framework. The latter will allow to simulate realistic power networks and consider the provision of more ancillary services such as local voltage support. Furthermore, in this paper the focus was on the interactions between the aggregators and the grid/market operators. Future work, will also address the settlement mechanism between the aggregators and their associated system users. Some considerations are that all users associated with an aggregator should have a natural incentive to participate in the aggregation entity. The settlement mechanism should allocate the aggregate costs and benefits in a fair way. Wholesale energy costs for each user can be calculated on the basis of their day-ahead energy schedules, whereas imbalances and flexibility contributions from individual users can be aggregated and represented as a percentage of the aggregate volumes. Subsequently, the individual costs and benefits of each user can be allocated on the basis of participation coefficients established in terms of energetic contributions.

\section{Acknowledgement}

The original ideas that led to the framework described in this paper were first conceptualised under the guidance of Professor Wil L. Kling (1950-2015). We would like to acknowledge his support in the development of these ideas, and his innovative vision in the energy management of distributed resources in modern power systems.

\section{References}

[1] CEN-CENELEC-ETSI Smart Grid Coordination Group, "Sustainable Processes," Nov. 2012.

[2] I. Lampropoulos, W.L. Kling, P.F. Ribeiro, J. van den Berg, History of demand side management and classification of demand response control schemes, in: Proc. of the IEEE PES General Meeting, Vancouver, Canada, 2013.

[3] Y. Zong, D. Kullmann, A. Thavlov, O. Gehrke, H.W. Bindner, Application of model predictive control for active load management in a distributed power system with high wind penetration, IEEE Trans. Smart Grid 3 (2) (2012) 10551062 . 
[4] S. Deilami, A.S. Masoum, P.S. Moses, M.A.S. Masoum, Real-time coordination of plug-in electric vehicle charging in smart grids to minimize power losses and improve voltage profile, IEEE Trans. Smart Grid 2 (3) (2011) 456-467.

[5] I. Lampropoulos, Energy Management of Distributed Resources in Power Systems Operations, Technische Universiteit Eindhoven, 2014.

[6] J.A. Short, D.G. Infield, L.L. Freris, Stabilization of grid frequency through dynamic demand control, IEEE Trans. Power Syst. 22 (3) (2007) 1284-1293.

[7] S.A. Pourmousavi, M.H. Nehrir, Real-time central demand response for primary frequency regulation in microgrids, IEEE Trans. Smart Grid 3 (4) (2012) 1988-1996.

[8] F.C. Schweppe, R.D. Tabors, J.L. Kirtley, H.R. Outhred, F.H. Pickel, A.J. Cox, Homeostatic utility control, IEEE Trans. Power Appl. Syst. PAS-99 (3) (1980) $1151-1163$.

[9] I. Lampropoulos, G.M.A. Vanalme, W.L. Kling, A methodology for modeling the behavior of electricity prosumers within the smart grid, in: IEEE PES Innovative Smart Grid Technologies Conference Europe, Gothenburg, 2010.

[10] A.J. Conejo, J.M. Morales, L. Baringo, Real-time demand response model, IEEE Trans. Smart Grid 1 (3) (2010) 236-242.

[11] C. De Jonghe, B.F. Hobbs, R. Belmans, Optimal generation mix with short-term demand response and wind penetration, IEEE Trans. Power Syst. 27 (2) (2012) 830-839.

[12] P. Palensky, D. Dietrich, Demand side management: Demand response, intelligent energy systems, and smart loads, IEEE Trans. Ind. Inf. 7 (3) (2011) 381-388.

[13] S.D. McArthur, E.M. Davidson, V.M. Catterson, A.L. Dimeas, N.D. Hatziargyriou, F. Ponci, T. Funabashi, Multi-agent systems for power engineering applications-Part I: concepts, approaches, and technical challenges, IEEE Trans. Power Syst. 22 (4) (2007) 1743-1752.

[14] D.S. Callaway, I.A. Hiskens, Achieving controllability of electric loads, Proc. IEEE 99 (1) (2011) 184-199.

[15] F.C. Schweppe, S.K. Mitter, Hierarchical system theory and electric power systems, in: Symposium on Real Time Control of Electric Power Systems, Baden, 1972.

[16] R.J. Bessa, M.A. Matos, The role of an aggregator agent for EV in the electricity market, in: 7th Mediterranean Conference and Exhibition on Power Generation, Transmission, Distribution and Energy Conversion (MedPower2010), Agia Napa, 2010

[17] P. Sánchez-Martín, S. Lumbreras, A. Alberdi-Alén, Stochastic programming applied to EV charging points for energy and reserve service markets, IEEE Trans. Power Syst. 31 (1) (2016) 198-205.

[18] A. Roos, S.Ø. Ottesen, T.F. Bolkes, Modeling consumer flexibility of an aggregator participating in the wholesale power market and the regulation capacity market, Energy Procedia 58 (2014) 79-86.

[19] I. Lampropoulos, N. Baghină, W.L. Kling, P.F. Ribeiro, A predictive control scheme for real-time demand response applications, IEEE Trans. Smart Grid Special Sect. Real-Time Demand Response 4 (4) (2013) 2049-2060.

[20] G. Lipari, G. Del Rosario, C. Corchero, F. Ponci, A. Monti, A real-time commercial aggregator for distributed energy resources flexibility management, Sustainable Energy Grids Netw. 15 (2017) 63-75.

[21] A.N.M.M. Haque, P.H. Nguyen, F.W. Bliek, J.G. Slootweg, Demand response for real-time congestion management incorporating dynamic thermal overloading cost, Sustainable Energy Grids Netw. 10 (2017) 65-74.

[22] A. Zecchino, K. Knezović, M. Marinelli, Identification of conflicts between transmission and distribution system operators when acquiring ancillary services from electric vehicles, in: IEEE PES Innovative Smart Grid Technologies Conference Europe, Torino, Italy, 2017.

[23] E.C., COMMISSION REGULATION (EU) 2017/1485 of 2 August 2017 establishing a guideline on electricity transmission system operation. Official Journal of the European Union, pp. 109-110, 25 Aug. 2017.
[24] I. Lampropoulos, M. van den Broek, W. van Sark, E. van der Hoofd, K. Hommes, Enabling flexibility from demand-side resources through aggregator companies, in: Smart Cities in the Mediterranean: Coping with Sustainability Objectives in Small and Medium-sized Cities and Island Communities, Springer International Publishing, Cham, Switzerland, 2017, pp. 333-353.

[25] I. Lampropoulos, P. Garoufalis, P.P. van den Bosch, W.L. Kling, Hierarchica predictive control scheme for distributed energy storage integrated with residential demand and photovoltaic generati, IET Gener. Transm. Distrib. 9 (15) (2015) 2319-2327.

[26] T. AlSkaif, A.C. Luna, M.G. Zapata, J.M. Guerrero, B. Bellalta, Reputation-based joint scheduling of households appliances and storage in a microgrid with a shared battery, Energy Build. 138 (2017) 228-239.

[27] S. van der Stelt, T. AlSkaif, W. van Sark, Techno-economic analysis of household and community energy storage for residential prosumers with smart appliances, Appl. Energy 209 (2018) 266-276.

[28] M. Heleno, M.A. Matos, J.P. Lopes, Availability and flexibility of loads for the provision of reserve, IEEE Trans. Smart Grid 6 (2) (2015) 667-674.

[29] B.V. TenneT TSO, "The Imbalance Pricing System," 1 Oct. 2016. [Online]. Available: https://www.tennet.eu/fileadmin/user upload/Company/Publications Technical_Publications/Dutch/imbalanceprice_3.6_clean_.doc.pdf. [Accessed 01.11.17].

[30] A.G.R. Vaz, B. Elsinga, W.G.J.H. van Sark, An artificial neural network to assess the impact of neighbouring photovoltaic systems in power forecasting in Utrecht, the Netherlands, Renew. Energy 85 (2016) 631-641.

[31] B. Elsinga, W.G.J.H.M. van Sark, Short-term peer-to-peer solar forecasting in a network of photovoltaic systems, Appl. Energy 206 (2017) 1464-1483.

[32] AF-Mercados, REF-E and Indra, Study on tariff design for distribution systems, Prepared for: DIRECTORATE-GENERAL FOR ENERGY, DIRECTORATE B - Internal Energy Market, Jan. 2015

[33] Tesla, "Powerall 2," Tesla, 2017. [Online]. Available: https://www.tesla.com/ nl_NL/powerwall? redirect=no [Accessed 01.11.17].

[34] I. Lampropoulos, M. van den Broek, E. van der Hoofd, K. Hommes, W. van Sark, A system perspective to the deployment of flexibility through aggregator companies in the Netherlands, Energy Policy 118 (2018) 534-551.

[35] EPEX Netherlands, "Day-Ahead Auction," EPEXSPOT, 2018. [Online]. Available: https://www.apxgroup.com/trading-clearing/day-ahead-auction/ [Accessed 25.06.17].

[36] J.P. Iria, F.J. Soares, M.A. Matos, Trading small prosumers flexibility in the energy and tertiary reserve markets, IEEE Trans. Smart Grid (2018) http: //dx.doi.org/10.1109/TSG.2018.2797001, (in press).

[37] N. Good, P. Mancarella, Flexibility in multi-energy communities with electrical and thermal storage: A stochastic, robust approach for multi-service demand response, IEEE Trans. Smart Grid 10 (1) (2019) 503-513, http://dx. doi.org/10.1109/TSG.2017.2745559.

[38] C. Dromacque, R. Grigoriou, Household Energy Price Index for Europe, VaasaETT Global Energy Think Tank, 2017.

[39] B.V. TenneT TSO, "Data export: Balance delta with prices, ” TenneT, 2018. [Online]. Available: http://www.tennet.org/english/operational_management/ export_data.aspx [Accessed 01.01.18].

[40] J.W. Taylor, P.E. McSharry, Short-term load forecasting methods: An evaluation based on European data, IEEE Trans. Power Syst. 22 (4) (2007) 2213 2219.

41] A. Yona, T. Senjyu, A.Y. Saber, T. Funabashi, H. Sekine, C.-H. Kim, Application of neural network to one-day-ahead $24 \mathrm{~h}$ generating power forecasting for photovoltaic system, in: International Conference on Intelligent Systems Applications To Power Systems, Toki Messe, Niigata, 2007.

[42] M. Ding, L. Wang, R. Bi, An ANN-based approach for forecasting the power output of photovoltaic system, Procedia Environ. Sci. 11 (2011) 1308-1315.

[43] MATLAB (Matrix Laboratory);, "Numerical Computing Environment and Programming Language, " Mathworks, 2017. [Online]. Available: http://www. mathworks.nl/products/matlab. [Accessed Oct. 2017]. 